\title{
Efecto manada en sectores económicos de las bolsas latinoamericanas: una visión pre y poscrisis subprime
}

\author{
Herding effect in economic sectors of the Latin American stock markets: \\ A pre and post-subprime crisis vision \\ Juan Benjamín Duarte Duarte ${ }^{\mathrm{a}, *}$, Laura Daniela Garcés Carreño ${ }^{a}$ \\ y Katherine Julieth Sierra Suárez ${ }^{b}$ \\ a Universidad Industrial de Santander, Santander, Colombia \\ b Universidad de Santander, Santander, Colombia
}

Recibido el 6 de mayo de 2015; aceptado el 28 de octubre de 2015

Disponible en Internet el 29 de enero de 2016

\begin{abstract}
Resumen
En los últimos años, el comportamiento de los agentes y lo que los motiva a tomar sus decisiones de inversión ha sido foco de estudio de muchos investigadores en las ramas de economía, finanzas y afines. Teniendo en cuenta esto, en este artículo se busca comprobar empíricamente uno de estos comportamientos, el efecto manada, mediante los modelos propuestos por Christie y Huang (1995) y Chang, Cheng y Khorana (2000), en el índice más representativo y en los sectores que lo componen, de los principales mercados de América Latina (Brasil, México, Chile, Colombia, Perú y Argentina) para el periodo 2002-2014, así como en los subperiodos pre y poscrisis originados por las hipotecas subprime. Los hallazgos de esta investigación muestran que el efecto manada está presente en el índice más representativo del mercado bursátil de Colombia, Chile y Perú y en algunos de los sectores que lo componen, ya sea en el periodo total y/o en los subperiodos de pre y poscrisis; en el mercado de Brasil, el efecto está presente en el sector bancario en el periodo total y en el subperiodo de precrisis; en el mercado de Argentina tal efecto se presentó en el sector bancario y en el sector de petróleo y gas, y en el mercado de México no existe ninguna evidencia de dicho efecto.
\end{abstract}

* Autor para correspondencia.

Correo electrónico: jbduarted@hotmail.com (J.B. Duarte Duarte).

La revisión por pares es responsabilidad de la Universidad Nacional Autónoma de México. 
Derechos Reservados (C) 2015 Universidad Nacional Autónoma de México, Facultad de Contaduría y Administración. Este es un artículo de acceso abierto distribuido bajo los términos de la Licencia Creative Commons CC BY-NC-ND 4.0.

Códigos JEL: C31; G14; G15

Palabras clave: Efecto manada; Mercados latinoamericanos; Dispersión de sección cruzada de los retornos

\begin{abstract}
In recent years the behavior of the agents and what motivates them to make their investment decisions; it has been the focus of study by many researchers in the fields of economics, finance and related fields. With this in mind, this article seeks to test empirically one of these behaviors, the herd effect, using the models proposed by Christie y Huang (1995) and Chang, Cheng y Khorana (2000), in the most representative index and the sectors that compose it, of the main markets in Latin America (Brazil, Mexico, Chile, Colombia, Peru and Argentina) in the period 2002-2014, as well as in the sub periods pre and post crisis caused by the subprime mortgage. The findings of this research show that the herd effect is present in the most representative stock index in Colombia, Chile and Peru and in some of the sectors that comprise it, either in the total period and/or in sub periods pre and post crisis; in the Brazilian stock market, the effect is present in the banking sector in the total period and in the sub period of pre-crisis; Argentina stock market that effect is present in the banking sector and in the oil and gas sector, and Mexico stock market there is no evidence of this effect. All Rights Reserved () 2015 Universidad Nacional Autónoma de México, Facultad de Contaduría y Administración. This is an open access item distributed under the Creative Commons CC License BY-NC-ND 4.0.
\end{abstract}

JEL classification: C31; G14; G15

Keywords: Herd effect; Latin America stock markets; Cross sectional dispersion of returns

\title{
Introducción
}

El comportamiento de los inversores en los últimos años y los modelos para predecirlos han sido foco de estudio por parte de muchos investigadores de diferentes disciplinas. De acuerdo con la hipótesis de mercados eficientes enunciada por Fama (1970), los inversores se comportan de manera racional y, por tanto, las diferentes estimaciones del precio de mercado de un activo reflejan toda la información disponible y deberían estar alrededor de su verdadero valor teórico; sin embargo, existe un nuevo enfoque, denominado finanzas conductuales, que nace por la necesidad de explicar determinados comportamientos de los participantes del mercado financiero que exponen una posible debilidad en las teorías de mercados eficientes. Esta disciplina es defendida por Shiller (2003), quien manifiesta que los inversores toman sus decisiones impulsados por aspectos psicológicos y sociológicos, propios de la naturaleza humana.

Uno de estos comportamientos es el efecto manada, que surge porque algunos inversores consideran como válidas las ideas o razonamientos de la mayoría, que en muchos casos pueden ser correctos y en otros erróneos; esto puede ocasionar razonamientos no válidos y sesgos en la toma de decisiones. Este comportamiento se puede dar de manera espontánea, cuando el inversor se deja llevar por sus impulsos naturales sin consideraciones dictadas por la razón; o de forma premeditada, cuando el inversor reflexiona sobre la toma de la decisión antes de llevarla a cabo.

A pesar de que en los trabajos consultados se hallan algunas investigaciones que han comprobado empíricamente la presencia de comportamiento manada en Latinoamérica, como la realizada por De Almeida, Costa y da Costa (2012), no se encuentra un estudio que evalúe dicho efecto por sectores económicos en los subperiodos pre y poscrisis subprime para los diferentes 
mercados bursátiles de la región. Por lo tanto, la presente investigación tiene como fin verificar la existencia del comportamiento manada en los principales mercados de América Latina (Brasil, México, Chile, Colombia, Perú y Argentina), así como también en los sectores que componen dichos mercados para los subperiodos antes y después de la crisis del 2008, resultados que podrían contribuir a reconocer el comportamiento de los inversionistas en periodos de rupturas bursátiles, que al ser interiorizados por los agentes, conllevaría la posible desaparición de retornos anormales y generaría que los mercados en el futuro tiendan a ser más eficientes (Kristjanpoller y Arenas, 2015).

Para realizar dicha comprobación se hace inicialmente un análisis preliminar estadístico y se utilizan el «modelo matemático I», propuesto por Christie y Huang (1995), y el «modelo II», desarrollado por Chang, Cheng y Khorana (2000). En ambos modelos la variable objeto de estudio es la dispersión de la rentabilidad logarítmica, que evalúa que tan cerca están los retornos individuales del retorno del mercado. El presente estudio se enfoca en estudiar los subperiodos pre y poscrisis en el intervalo de 2002 a 2014.

Los resultados obtenidos al utilizar la metodología propuesta muestran que mediante el modelo I no se halló evidencia alguna de efecto manada ni en los subperiodos pre y poscrisis, ni en el periodo total (2002-2014); sin embargo, al utilizar el modelo II se detectó la presencia de dicho efecto en algunos países de Latinoamérica — principalmente en Colombia, Chile y Perú- en algunos sectores y subperiodos de estudio. Hallazgos que son importantes, dado que permiten identificar la existencia de comportamientos por parte de los inversores que no son consistentes con la hipótesis de eficiencia de Fama. Lo anterior es significativo porque podría contribuir a comprender la dinámica del mercado desde un punto de vista humano y generar nuevos temas de investigación buscando modelos de predicción de rentabilidades incluyendo factores conductuales. Adicionalmente, estos resultados son relevantes para los inversores que administran portafolios con activos de la región, teniendo en cuenta que si existe el efecto manada los portafolios que elijan deben ser más diversificados.

Este artículo se compone de la siguiente manera. En primer lugar se lleva a cabo una revisión de la literatura para establecer a qué hace referencia el término «efecto manada», los modelos matemáticos que se usan para comprobarlo y los principales estudios que se han llevado a cabo en el mundo; luego se exponen los datos y la metodología a utilizar; seguido se aplica la metodología y se obtienen los resultados, y por último se finaliza el estudio con las principales conclusiones.

\section{Revisión de la literatura}

El efecto manada ha sido estudiado y documentado por un gran número de investigadores en el área financiera y afines, lo que ha dado como resultado una variedad de definiciones. Borensztein y Gelos (2003) enuncian que este efecto consiste en inversores que compran y venden activos solo porque observan a otros hacerlo. Hott (2009) define este efecto como la influencia positiva de las decisiones de unos inversores sobre las decisiones de un inversor en particular. Por último Bikhchandani y Sharma, 2001 enuncian que el efecto manada es el intento obvio de un inversor de copiar el comportamiento de otros inversores, matizando que existe una diferencia entre la conducta intencional y espuria. La primera se da por la intención de uno o más agentes de imitar a sus similares, por razones asociadas a falta de información, estructuras de compensación y reputación. La segunda corresponde a agentes que se enfrentan a situaciones y conjuntos de información similares, que los llevan a tomar decisiones equivalentes sin que se presente influencia de un agente sobre otro, es decir, el comportamiento se evidencia porque la interpretación de la información coincide. 
De acuerdo con la literatura científica, dependiendo del comportamiento de los inversores, el efecto manada se puede abordar desde 2 dimensiones: racional e irracional. La manada racional, de acuerdo con Scharfstein y Stein (1990), hace referencia al hecho de tomar decisiones utilizando toda la información disponible de una manera eficiente; además, Devenow y Welch (1996) manifiestan que esta se puede dar por 3 efectos: a) externalidades en las ganancias, cuando los costos o las ganancias de un agente se ven afectados por el número de agentes que toman la decisión; b) por problemas del agente-principal, que muestran que los directivos, buscando preservar o ganar reputación, pueden preferir esconderse en la manada, imitando las acciones de sus similares e ignorar su información privada, para así evitar ser evaluados individualmente, o liderar la manada, demostrando su calidad frente al resto, y c) las externalidades en la información, cuando las decisiones de los agentes se basan en adquirir información útil de las decisiones de los agentes anteriores y actuar de la misma manera ignorando racionalmente su propia información. Por otro lado, en la dimensión irracional, Ortiz, Sarto y Vicente (2013) sostienen que surge de la psicología del inversor, ya que sus decisiones están influenciadas por sesgos cognitivos, imposibles de pasar por alto por los seres humanos, es decir, que se presenta una distorsión en el modo en que las personas perciben la realidad, lo que hace que en un momento dado tomen una decisión de inversión sin un sustento fundamental.

Dado que este comportamiento tiene implicaciones sobre el movimiento del precio de los activos y puede afectar sus características de riesgo y rentabilidad, muchos investigadores están interesados en comprobar su existencia empíricamente mediante modelos matemáticos. Algunos de estos modelos usan como variable de estudio la dispersión y evalúan el efecto manada a nivel del mercado en general, sin tener en cuenta el comportamiento individual del inversor. Teniendo en cuenta lo anterior, Christie y Huang (1995) propusieron un modelo tomando como medida de dispersión de los retornos la desviación estándar de sección cruzada (cross sectional standard deviation [CSSD]), esto con el fin estudiar las fuertes caídas y subidas del precio de los activos en el mercado de valores de Estados Unidos y los sectores que lo componen, en el periodo 19251988, y de acuerdo con sus hallazgos no se puede concluir evidencia a favor de la presencia de dicho efecto. A partir de este planteamiento, Chang et al. (2000) formularon un modelo más sensible usando la desviación absoluta de sección cruzada (cross sectional absolute deviation [CSAD]) para identificar la presencia de efecto manada en los mercados de Estados Unidos, Hong Kong, Corea del Sur y Taiwán; el estudio abarca el periodo de 1963 a 1997, y sus resultados indican evidencia significativa de efecto manada en los mercados de Corea del Sur y Taiwán, evidencia parcial en el mercado de Hong Kong y ninguna evidencia en el mercado de Estados Unidos. Basados en estos 2 modelos, algunos investigadores han examinado la existencia de efecto manada en diferentes mercados bursátiles del mundo, en periodos de normalidad y considerando la denominada crisis subprime, que fue un fenómeno de riesgo sistemático que se originó en el mercado estadounidense para luego trasladarse a los mercados financieros globales (AMV, 2009).

Entre los estudios más relevantes destacan: en el mercado europeo, Henker, Henker y Mitsios (2006) analizaron la presencia de efecto manada en las 160 empresas de mayor actividad y capitalización bursátil que componen el índice ASX200, así como en los 10 sectores a los cuales fueron asignadas, considerando precios diarios e intra-día en el periodo 2001-2002, concluyendo que no existe evidencia de efecto manada, lo que indica que la información es difundida eficientemente para la toma de decisiones de los inversores de la bolsa de valores de Australia. Por su parte, basados en datos de precios diarios, semanales y mensuales, Caporale, Economou y Philippas (2008) examinaron la existencia de efecto manada en extremas condiciones de mercado en la bolsa de valores de Atenas en el periodo 1998-2007; sus resultados evidencian que la presencia de efecto es más fuerte, considerando datos diarios, lo que revela que el fenómeno se presenta en 
el corto plazo. Además, Ohlson (2010) encontró que en general el comportamiento manada está presente en el mercado de valores de Estocolmo en el periodo de 1998 a 2009, mostrando mayor presencia en los mercados alcistas y en los años 2005 y 2007. Asimismo, Economou, Kostakis y Philippas (2011) hallaron, durante el periodo 1998-2008, efecto manada en los mercados de Grecia e Italia, efecto parcial en Portugal y ninguna evidencia de este en España; además, el efecto presentaba asimetrías significativas tanto con los precios de los activos a la alza y a la baja como en días de baja y alta actividad de negociación y volatilidad. Posteriormente, Ouarda, Bouri, y Bernard (2013) estudiaron la existencia de efecto manada en 10 industrias compuestas por las 174 empresas de mayor capitalización bursátil del EuroStoxx600, con precios mensuales en el periodo desde enero de 1998 hasta diciembre de 2010. Los resultados muestran que, exceptuando el sector de bienes de consumo, el efecto está presente en todos los sectores. Al mismo tiempo, la existencia del efecto se da en todos los sectores durante meses caracterizados por fuerte volatilidad. Además los autores consideran que durante la crisis financiera mundial 2007-2008 y la crisis asiática, el comportamiento manada es evidenciado.

En el mercado asiático, Demirer y Kutan (2006) analizan la existencia de efecto manada en el mercado de China, en periodos de inusuales movimientos del mercado comprendidos entre 1999 y 2002, tanto a nivel del mercado total como a nivel sectorial (los autores definieron 18 grupos de industrias). Además, realizaron un análisis para las bolsas de valores de Shanghai y Shenzhen por separado, en el periodo de mayo de 1993 a noviembre de 2001, en el total del mercado y en los sectores que los componen; sus hallazgos no indican presencia de efecto manada en el mercado de China, pero observaron que la dispersión de los retornos era más baja cuando la rentabilidad de las acciones se encontraba en la cola inferior de la distribución, lo que sugeriría que el comportamiento de los inversores es más similar durante caída del precio de los activos. En un estudio similar, Tan, Chiang, Mason y Nelling (2008) analizaron el comportamiento manada en China de las acciones tipo A (dominadas por los inversores locales) y tipo B (dominadas por los inversores extranjeros) de las bolsas de valores de Shenzhen y Shanghai desde 1994 a 2003; sus resultados, basados en datos diarios, muestran fuerte presencia de efecto manada, mientras que con datos semanales y mensuales el efecto es más débil, lo que sugiere que dicho efecto es más evidente en horizontes de tiempo cortos; en general, el efecto está presente en los 2 tipos de acciones para periodos de alza, baja, volumen de transacción y volatilidad altos. Adicional a esto, Lao y Singh (2011) estudiaron el efecto en las 300 firmas de mayor capitalización bursátil que componen el Shanghai A-Share índex y el Bombay Stock Exchange índex, que corresponden a los índices más representativos de China e India, respectivamente; el periodo que analizaron fue de julio de 1999 a junio de 2009. Los autores encontraron que el comportamiento manada en India se presenta cuando los precios de los activos aumentan, mientras que en China predomina cuando el mercado está en declive y el volumen de transacción es alto. Recientemente, Liu (2013) realizó un análisis empírico, con datos de precios diarios, de los activos de mayor negociación y que en conjunto forman el $60 \%$ de la capitalización bursátil total del mercado, en el periodo 2002-2012, para comprobar la existencia de efecto manada en el mercado de Singapur; sus resultados manifiestan que el comportamiento manada es más significativo cuando el mercado está en descenso, durante periodos de crisis financieras y en los sectores financieros cuidado de la salud y servicios de consumo.

En el mercado latinoamericano, De Almeida et al. (2012) seleccionaron las acciones de estudio, con base a la composición de los principales índices de los mercados de la región, para comprobar si existía efecto manada. Los resultados no detectaron este fenómeno en la mayoría de mercados latinoamericanos; sin embargo, en el mercado chileno entre 2000 y 2010 sí se detectó el efecto en activos de alto volumen de negociación, alta y baja volatilidad. 
A nivel global, Chiang y Zheng (2010) examinan el comportamiento manada con datos diarios de 18 países, divididos en mercados desarrollados, latinoamericanos y asiáticos en el periodo de 1998 a 2009; de acuerdo con sus resultados, exceptuando los mercados de Latinoamérica y el de Estados Unidos, el efecto está presente tanto en subidas como en caídas del precio de la acción. Además, la evidencia sugiere que el efecto manada se presenta en los países de Estados Unidos y Latinoamérica cuando estos se encuentran en crisis. Posteriormente, Chen (2013) realizó un estudio en el periodo 2000-2009 con 69 países, los cuales clasificó en desarrollados, emergentes y de frontera; sus conclusiones sugieren fuerte evidencia de efecto manada en la mayoría de mercados bursátiles, destacando que en los mercados desarrollados esta conducta es más pronunciada, por la facilidad de acceso a la información que conduce a consensos en el mercado; asimismo concluye que, en general, los inversores tienden a manifestar este comportamiento como respuesta a las malas noticias. Además, Gebka y Wohar (2013) comprobaron si se presentaba efecto manada en la muestra total y en 9 sectores globales de 32 países, en el periodo de 1998 a 2012, hallando resultados que indican presencia de efecto en algunos sectores, como petróleo y gas, materiales básicos y servicio al consumidor.

\section{Metodología y datos}

\section{Datos}

Los datos utilizados en la presente investigación corresponden a los precios de cierre diario de las empresas que componen el índice más representativo de cada una de las principales bolsas de valores de Latinoamérica (Brasil, México, Chile, Colombia, Perú y Argentina) durante el periodo comprendido entre el 2 de enero de 2002 y el 30 de junio de 2014. Los criterios de selección para tener en cuenta una empresa en su respectivo sector son: omitir los días en los cuales la bolsa de valores de cada país no operó; elegir las empresas que pertenecían a la canasta del segundo trimestre del 2014, excluyendo las empresas con un número de cotizaciones inferiores a un año; sin embargo, en el caso particular del mercado accionario de Colombia se flexibilizó este criterio debido a su poca dinámica, tomando aquellas empresas que han estado listadas por lo menos una vez en las canastas trimestrales del COLCAP desde su entrada en vigencia en 2008, que estén cotizando a junio de 2014 y que posean un nivel de liquidez alto según criterio de la Bolsa de Valores de Colombia. Los datos se obtuvieron de Bloomberg Professional Service.

El efecto se analiza para cada mercado bursátil latinoamericano, usando todos los activos seleccionados y para los sectores que los componen de acuerdo con la clasificación propuesta por cada una de las respectivas bolsas de valores, por tal motivo los sectores difieren de un país a otro; esto se hizo con el fin de facilitar a los inversores e investigadores de cada país el reconocimiento de las empresas de acuerdo al sector en el que se encuentre. Además, con el objetivo de conocer el impacto que tuvo el periodo de pre y poscrisis originadas por las subprime, sobre el comportamiento de los inversores, se seleccionó como fecha crítica el 10 de octubre de 2008 para dividir los periodos pre y poscrisis, considerando que este día se produjo una caída histórica de las cotizaciones bursátiles de la mayoría de bolsas del mundo.

\section{Modelo I}

Propuesto por Christie y Huang (1995), se enfoca en evaluar la presencia de efecto manada durante momentos de extremos movimientos del mercado.

La variable objeto de estudio es la desviación estándar de sección cruzada (cross sectional standard deviation [CSSD]) de los retornos individuales con respecto al retorno del mercado, 
planteada en la ecuación 1.

$$
\operatorname{CSSD}_{t}=\sqrt{\frac{\sum_{i=1}^{N}\left(R_{i, t}-R_{m, t}\right)^{2}}{N-1}}
$$

donde $R_{i t}$ es la rentabilidad de la acción $i$ en el tiempo $t$ y $R_{m t}$ es el promedio de sección cruzada de las $\mathrm{N}$ rentabilidades de las acciones disponibles en el mercado en el tiempo $t$. Luego, para comprobar la existencia de efecto manada durante condiciones extremas del mercado se estima el modelo de regresión lineal binario, presentado en la ecuación 2.

$$
\operatorname{CSSD}_{t}=\alpha+\beta_{1} D^{L}+\beta_{2} D^{U}+\varepsilon_{t}
$$

donde $D^{L}$ toma el valor de 1 si el retorno del mercado en el tiempo $t$ se encuentra en el extremo inferior de la cola de la distribución, y cero de otra manera. Por otro lado, $D^{U}$ toma el valor de 1 si el retorno del mercado se encuentra en el extremo superior de la cola de la distribución y cero de otra manera. El coeficiente $\alpha$ expresa la dispersión promedio de la muestra, excluyendo las 2 regiones cubiertas por las variables dicotómicas.

Como la desviación estándar de sección cruzada (CSSD) puede estar afectada por valores atípicos, tanto Christie y Huang (1995) como Chang et al. (2000) definen la desviación absoluta de sección cruzada (cross sectional absolute deviation [CSAD]) como una mejor medida de dispersión (ecuación 3).

$$
C S A D_{t}=\frac{\sum_{i=1}^{N}\left|R_{i, t}-R_{m, t}\right|}{N}
$$

donde $R_{i t}$ y $R_{m t}$ equivalen a lo definido en CSSD (ecuación 1).

Teniendo en cuenta lo anterior, en el presente trabajo se estima la regresión de la ecuación 2 , usando como variable objeto de estudio la desviación absoluta de sección cruzada (CSAD), como se indica en la ecuación 4.

$$
C S A D_{t}=\alpha+\beta_{1} D^{L}+\beta_{2} D^{U}+\varepsilon_{t}
$$

De acuerdo con este modelo, la presencia de negatividad y significancia estadística en $\beta_{1}$ y $\beta_{2}$ indicarían una disminución en promedio de la dispersión, sugiriendo la presencia de efecto manada.

\section{Modelo II}

Chang et al. (2000), en base a los supuestos del modelo CAPM, demostraron que existe una relación creciente y lineal entre la dispersión calculada por CSAD y el retorno del mercado $\left(R_{m t}\right)$, y propusieron una prueba alternativa para detectar la presencia de efecto manada, que requiere un término adicional en la regresión que capture alguna posible relación no lineal entre la dispersión y el retorno del mercado. De acuerdo con lo anterior, plantearon la ecuación 5:

$$
C S A D_{t}=\alpha+\gamma_{1}\left|R_{m, t}\right|+\gamma_{2} R_{m, t}^{2}+\varepsilon_{t}
$$

donde CSAD se define en la ecuación 3 y $R_{m, t}$ es el retorno promedio de las $\mathrm{N}$ acciones disponibles en el mercado en el tiempo $t$.

Bajo los supuestos de los modelos de valoración de activos racionales, se espera que $\gamma_{1}$ sea positiva. Sin embargo, para establecer la presencia de efecto manada se requiere un coeficiente $\gamma_{2}$ 
negativo y estadísticamente significativo, indicando que después de un movimiento en el mercado $C S A D_{t}$ decrece o incrementa a una tasa decreciente.

Como existe la posibilidad de que el grado de efecto manada pueda darse cuando el mercado está perdiendo (down) o ganando (up), Chang et al. (2000) estimaron las regresiones 29 y 30.

$$
\begin{aligned}
& C S A D_{t}^{D O W N}=\alpha+\gamma_{1}^{D O W N}\left|R_{m, t}^{D O W N}\right|+\gamma_{2}^{D O W N}\left(R_{m, t}^{D O W N}\right)^{2}+\varepsilon_{t} \\
& C S A D_{t}^{U P}=\alpha+\gamma_{1}^{U P}\left|R_{m, t}^{U P}\right|+\gamma_{2}^{U P}\left(R_{m, t}^{U P}\right)^{2}+\varepsilon_{t}
\end{aligned}
$$

donde $R_{m, t}^{D O W N}$ y $R_{m, t}^{U P}$ son el retorno promedio de las $\mathrm{N}$ acciones disponibles en el mercado en el tiempo $t$ cuando el retorno es negativo o positivo, respectivamente. Se hace uso del valor absoluto para facilitar la comparación de los coeficientes que acompañan el término lineal.

\section{Resultados}

\section{Estadísticas descriptivas}

En cuanto a las series de rentabilidades, al comparar las medias en los subperiodos de precrisis y poscrisis se observa que este estadístico es consistentemente más alto en el subperiodo posterior a la crisis en todos los países. Así mismo, al analizar la desviación estándar se encuentra que la dispersión de los datos es más alta en los años posteriores a 2008. En cuanto al sesgo, se observa que su valor es diferente de cero y que su comportamiento varía entre los distintos mercados; en particular se halla que en los países de Brasil, México y Chile el sesgo tiende a ser mayor en subperiodos poscrisis que en subperiodos precrisis, lo cual indica que la probabilidad de obtener probabilidades por encima de la media disminuye después de la crisis subprime, mientras que en Colombia, Perú y Argentina el comportamiento del sesgo en los subperiodos es inverso. Finalmente, en las estimaciones de la curtosis se encuentra que en el subperiodo de poscrisis los datos se concentran mayormente alrededor de la media, disminuyendo la probabilidad de grandes pérdidas o ganancias. Por otra parte, al analizar los estadísticos descriptivos de la variable CSAD se encuentra que en el subperiodo poscrisis todos los estadísticos tienen un valor positivo mayor que los estimados en el subperiodo precrisis. Adicionalmente se identifica un comportamiento particular de los estadísticos del sector servicios varios de Chile con un alto sesgo positivo y leptocurtosis tanto en la serie de rentabilidades como en la de CSAD (tabla 1). A nivel general, en ambas series se rechaza la hipótesis de normalidad mediante la evaluación del estadístico Jarque Bera, cuya probabilidad es cero, rechazando la hipótesis de normalidad para cada variable. Sin embargo, hay que tener en cuenta que en muestras grandes de datos, según Gujarati y Porter (2010, pág. 99), se puede flexibilizar la exigencia de normalidad en los modelos de regresión lineal.

\section{Modelo I}

Para comprobar la existencia de efecto manada en las empresas que componen el índice más representativo de los mercados accionarios de América Latina y los sectores al interior de este, inicialmente se empleó el modelo de regresión de variables dummy propuesto por Christie y Huang $(\mathrm{CH})$ en 1995, considerando como medida de dispersión CSAD, tal como se plantea en la ecuación 4. Esta ecuación es estimada usando como criterios extremos el 1, el 5 y el 10\% por debajo y por encima de la distribución del retorno del mercado, para los subperiodos de precrisis y poscrisis y para el periodo total. 
Estadísticos descriptivos: Rentabilidad y CSAD

\begin{tabular}{|c|c|c|c|c|c|c|c|c|c|c|c|c|c|c|c|c|c|c|c|}
\hline \multirow[t]{2}{*}{ Sector } & & \multicolumn{9}{|c|}{ Precrisis } & \multicolumn{9}{|c|}{ Poscrisis } \\
\hline & & $\bar{x}$ & $\tilde{x}$ & $\operatorname{Max}$ & Min & $\sigma$ & $S$ & $C$ & $P_{j b}$ & $N$ & $\bar{x}$ & $\tilde{x}$ & $\operatorname{Max}$ & Min & $\sigma$ & $S$ & $C$ & $P_{j b}$ & $N$ \\
\hline \multicolumn{20}{|l|}{ Brasil } \\
\hline \multirow[t]{2}{*}{ Mercado total } & $R$ & 0.001 & 0.001 & 0.10 & -0.10 & 0.016 & -0.42 & 6.04 & $0 \%$ & 1679 & 0.000 & 0.000 & 0.13 & -0.11 & 0.015 & 0.20 & 13.39 & $0 \%$ & 1414 \\
\hline & $C S A D$ & 0.016 & 0.016 & 0.07 & 0.01 & 0.005 & 2.66 & 20.87 & $0 \%$ & 1679 & 0.015 & 0.014 & 0.06 & 0.00 & 0.006 & 2.98 & 16.11 & $0 \%$ & 1414 \\
\hline \multirow[t]{2}{*}{ Bancos } & $R$ & 0.001 & 0.001 & 0.09 & -0.08 & 0.020 & 0.06 & 4.31 & $0 \%$ & 1681 & 0.000 & 0.001 & 0.20 & -0.12 & 0.020 & 0.56 & 13.27 & $0 \%$ & 1412 \\
\hline & $C S A D$ & 0.009 & 0.008 & 0.04 & 0.00 & 0.005 & 1.55 & 7.55 & $0 \%$ & 1681 & 0.007 & 0.006 & 0.07 & 0.00 & 0.004 & 3.57 & 34.37 & $0 \%$ & 1412 \\
\hline \multirow[t]{2}{*}{ Financiero } & $R$ & 0.001 & 0.001 & 0.12 & -0.12 & 0.019 & -0.22 & 5.99 & $0 \%$ & 1647 & 0.001 & 0.000 & 0.19 & -0.13 & 0.017 & 0.94 & 22.73 & $0 \%$ & 1411 \\
\hline & $C S A D$ & 0.012 & 0.010 & 0.08 & 0.00 & 0.010 & 1.55 & 7.36 & $0 \%$ & 1647 & 0.013 & 0.011 & 0.09 & 0.00 & 0.007 & 3.41 & 27.33 & $0 \%$ & 1411 \\
\hline \multirow[t]{2}{*}{ Constr. } & $R$ & 0.001 & 0.001 & 0.15 & -0.15 & 0.029 & 0.12 & 6.35 & $0 \%$ & 760 & 0.000 & 0.000 & 0.14 & -0.17 & 0.027 & -0.18 & 5.98 & $0 \%$ & 1411 \\
\hline & $C S A D$ & 0.018 & 0.017 & 0.09 & 0.00 & 0.011 & 2.00 & 10.73 & $0 \%$ & 760 & 0.015 & 0.013 & 0.09 & 0.00 & 0.008 & 2.33 & 12.75 & $0 \%$ & 1411 \\
\hline \multirow[t]{2}{*}{ Servicios públicos } & $R$ & 0.000 & 0.001 & 0.10 & -0.09 & 0.021 & -0.11 & 3.93 & $0 \%$ & 1681 & 0.000 & 0.000 & 0.12 & -0.08 & 0.014 & 0.47 & 11.93 & $0 \%$ & 1411 \\
\hline & $C S A D$ & 0.015 & 0.013 & 0.08 & 0.00 & 0.007 & 2.11 & 13.70 & $0 \%$ & 1681 & 0.012 & 0.010 & 0.07 & 0.00 & 0.006 & 3.17 & 21.19 & $0 \%$ & 1411 \\
\hline \multirow[t]{2}{*}{ Consumo cíclico } & $R$ & 0.002 & 0.001 & 0.54 & -0.36 & 0.039 & 2.34 & 60.22 & $0 \%$ & 960 & 0.001 & 0.001 & 0.16 & -0.11 & 0.016 & 0.54 & 12.41 & $0 \%$ & 1411 \\
\hline & $C S A D$ & 0.022 & 0.016 & 0.53 & 0.00 & 0.033 & 8.28 & 96.97 & $0 \%$ & 960 & 0.013 & 0.012 & 0.11 & 0.00 & 0.008 & 3.71 & 30.32 & $0 \%$ & 1411 \\
\hline \multirow[t]{2}{*}{ Consumo no cíclico } & $R$ & 0.000 & 0.001 & 0.07 & -0.07 & 0.016 & -0.31 & 4.82 & $0 \%$ & 1681 & 0.001 & 0.001 & 0.10 & -0.09 & 0.014 & -0.08 & 9.77 & $0 \%$ & 1411 \\
\hline & $C S A D$ & 0.014 & 0.013 & 0.07 & 0.00 & 0.007 & 1.41 & 7.87 & $0 \%$ & 1681 & 0.014 & 0.013 & 0.06 & 0.00 & 0.006 & 1.96 & 9.59 & $0 \%$ & 1411 \\
\hline \multirow[t]{2}{*}{ Madera, papel } & $R$ & -0.004 & -0.002 & 0.10 & -0.11 & 0.025 & -0.67 & 7.06 & $0 \%$ & 257 & 0.000 & 0.000 & 0.13 & -0.09 & 0.021 & 0.17 & 6.17 & $0 \%$ & 1409 \\
\hline & $C S A D$ & 0.012 & 0.009 & 0.07 & 0.00 & 0.011 & 1.82 & 7.78 & $0 \%$ & 257 & 0.011 & 0.009 & 0.18 & 0.00 & 0.009 & 5.37 & 80.71 & $0 \%$ & 1409 \\
\hline \multirow[t]{2}{*}{ Siderurgia } & $R$ & 0.001 & 0.002 & 0.12 & -0.15 & 0.024 & -0.36 & 5.06 & $0 \%$ & 1681 & 0.000 & -0.001 & 0.17 & -0.17 & 0.025 & 0.29 & 7.94 & $0 \%$ & 1411 \\
\hline & $C S A D$ & 0.010 & 0.009 & 0.08 & 0.00 & 0.007 & 2.35 & 15.10 & $0 \%$ & 1681 & 0.009 & 0.007 & 0.06 & 0.00 & 0.006 & 2.26 & 12.87 & $0 \%$ & 1411 \\
\hline \multirow[t]{2}{*}{ Transporte } & $R$ & 0.001 & 0.000 & 0.37 & -0.13 & 0.021 & 2.98 & 61.93 & $0 \%$ & 1562 & 0.000 & 0.000 & 0.14 & -0.15 & 0.018 & -0.08 & 14.20 & $0 \%$ & 1411 \\
\hline & $C S A D$ & 0.016 & 0.013 & 0.55 & 0.00 & 0.018 & 18.14 & 519.2 & $0 \%$ & 1562 & 0.014 & 0.013 & 0.08 & 0.00 & 0.009 & 2.19 & 11.77 & $0 \%$ & 1411 \\
\hline \multirow[t]{2}{*}{ Minería } & $R$ & 0.001 & 0.001 & 0.13 & -0.19 & 0.022 & -0.71 & 9.58 & $0 \%$ & 1678 & 0.000 & -0.001 & 0.58 & -0.16 & 0.031 & 4.95 & 94.67 & $0 \%$ & 1411 \\
\hline & $C S A D$ & 0.006 & 0.004 & 0.15 & 0.00 & 0.008 & 6.34 & 82.74 & $0 \%$ & 1678 & 0.014 & 0.009 & 0.79 & 0.00 & 0.026 & 19.93 & 575.1 & $0 \%$ & 1411 \\
\hline \multirow[t]{2}{*}{ Telec. } & $R$ & 0.000 & 0.000 & 0.11 & -0.10 & 0.022 & -0.10 & 4.19 & $0 \%$ & 1681 & 0.000 & 0.000 & 0.13 & -0.15 & 0.019 & 0.09 & 10.24 & $0 \%$ & 1411 \\
\hline & $C S A D$ & 0.013 & 0.011 & 0.09 & 0.00 & 0.009 & 2.14 & 12.54 & $0 \%$ & 1681 & 0.012 & 0.010 & 0.12 & 0.00 & 0.011 & 3.75 & 28.19 & $0 \%$ & 1411 \\
\hline \multirow[t]{2}{*}{ PQ } & $R$ & 0.001 & 0.002 & 0.10 & -0.09 & 0.020 & -0.26 & 4.68 & $0 \%$ & 1681 & 0.000 & 0.000 & 0.14 & -0.11 & 0.021 & 0.00 & 7.49 & $0 \%$ & 1411 \\
\hline & $C S A D$ & 0.010 & 0.008 & 0.08 & 0.00 & 0.008 & 1.69 & 8.18 & $0 \%$ & 1681 & 0.009 & 0.007 & 0.07 & 0.00 & 0.008 & 2.25 & 11.34 & $0 \%$ & 1411 \\
\hline \multicolumn{20}{|l|}{ México } \\
\hline \multirow[t]{2}{*}{ Mercado total } & $R$ & 0.001 & 0.002 & 0.04 & -0.08 & 0.011 & -0.93 & 7.61 & $0 \%$ & 1710 & 0.001 & 0.001 & 0.11 & -0.07 & 0.013 & 0.16 & 11.61 & $0 \%$ & 1436 \\
\hline & $C S A D$ & 0.013 & 0.012 & 0.07 & 0.00 & 0.005 & 2.83 & 23.66 & $0 \%$ & 1710 & 0.013 & 0.011 & 0.08 & 0.00 & 0.006 & 3.65 & 29.15 & $0 \%$ & 1436 \\
\hline \multirow[t]{2}{*}{ Bienes de consumo } & $R$ & 0.002 & 0.001 & 0.07 & -0.08 & 0.015 & -0.07 & 5.62 & $0 \%$ & 1410 & 0.001 & 0.001 & 0.14 & -0.09 & 0.017 & 0.63 & 10.68 & $0 \%$ & 1436 \\
\hline & $C S A D$ & 0.010 & 0.008 & 0.08 & 0.00 & 0.010 & 2.18 & 10.24 & $0 \%$ & 1410 & 0.011 & 0.008 & 0.12 & 0.00 & 0.010 & 3.18 & 21.41 & $0 \%$ & 1436 \\
\hline Materiales & $R$ & 0.001 & 0.002 & 0.10 & -0.14 & 0.017 & -0.74 & 9.12 & $0 \%$ & 1710 & 0.001 & 0.001 & 0.16 & -0.12 & 0.019 & 0.24 & 11.11 & $0 \%$ & 1436 \\
\hline & $C S A D$ & 0.014 & 0.012 & 0.19 & 0.00 & 0.011 & 5.17 & 66.48 & $0 \%$ & 1710 & 0.013 & 0.011 & 0.07 & 0.00 & 0.008 & 2.28 & 11.62 & $0 \%$ & 1436 \\
\hline A.B.T. & $R$ & 0.000 & 0.001 & 0.04 & -0.06 & 0.010 & -0.38 & 5.04 & $0 \%$ & 1710 & 0.001 & 0.001 & 0.06 & -0.15 & 0.013 & -1.52 & 19.09 & $0 \%$ & 1436 \\
\hline & $C S A D$ & 0.010 & 0.009 & 0.06 & 0.00 & 0.005 & 1.62 & 9.64 & $0 \%$ & 1710 & 0.011 & 0.009 & 0.30 & 0.00 & 0.011 & 15.10 & 388.7 & $0 \%$ & 1436 \\
\hline
\end{tabular}


Tabla 1 (Continuación)

\begin{tabular}{|c|c|c|c|c|c|c|c|c|c|c|c|c|c|c|c|c|c|c|c|}
\hline \multirow[t]{2}{*}{ Sector } & & \multicolumn{9}{|c|}{ Precrisis } & \multicolumn{9}{|c|}{ Poscrisis } \\
\hline & & $\bar{x}$ & $\tilde{x}$ & $\operatorname{Max}$ & Min & $\sigma$ & $S$ & $C$ & $P_{j b}$ & $N$ & $\bar{x}$ & $\tilde{x}$ & $\operatorname{Max}$ & Min & $\sigma$ & $S$ & $C$ & $P_{j b}$ & $N$ \\
\hline \multirow[t]{2}{*}{ Financiero } & $R$ & 0.001 & 0.001 & 0.10 & -0.18 & 0.018 & -0.71 & 11.93 & $0 \%$ & 1701 & 0.001 & 0.001 & 0.12 & -0.08 & 0.017 & 0.36 & 9.36 & $0 \%$ & 1436 \\
\hline & $C S A D$ & 0.011 & 0.008 & 0.22 & 0.00 & 0.013 & 5.62 & 63.45 & $0 \%$ & 1701 & 0.012 & 0.011 & 0.22 & 0.00 & 0.010 & 8.27 & 148.6 & $0 \%$ & 1436 \\
\hline \multirow[t]{2}{*}{ Consumo frecuente } & $R$ & 0.000 & 0.001 & 0.06 & -0.18 & 0.014 & -1.82 & 24.40 & $0 \%$ & 1710 & 0.001 & 0.001 & 0.14 & -0.08 & 0.015 & 1.26 & 18.15 & $0 \%$ & 1436 \\
\hline & $C S A D$ & 0.009 & 0.008 & 0.26 & 0.00 & 0.009 & 14.66 & 402.3 & $0 \%$ & 1710 & 0.010 & 0.008 & 0.20 & 0.00 & 0.010 & 8.01 & 123.6 & $0 \%$ & 1436 \\
\hline \multirow{2}{*}{ Industrial } & $R$ & 0.001 & 0.002 & 0.14 & -0.13 & 0.018 & 0.02 & 9.97 & $0 \%$ & 1703 & 0.001 & 0.001 & 0.14 & -0.08 & 0.014 & 0.34 & 13.72 & $0 \%$ & 1436 \\
\hline & $C S A D$ & 0.012 & 0.010 & 0.15 & 0.00 & 0.011 & 4.61 & 43.81 & $0 \%$ & 1703 & 0.012 & 0.010 & 0.09 & 0.00 & 0.007 & 2.98 & 22.94 & $0 \%$ & 1436 \\
\hline \multirow[t]{2}{*}{ Demás empresas } & $R$ & 0.001 & 0.001 & 0.08 & -0.06 & 0.017 & -0.02 & 4.36 & $0 \%$ & 1706 & 0.001 & 0.001 & 0.09 & -0.07 & 0.014 & 0.24 & 6.88 & $0 \%$ & 1436 \\
\hline & $C S A D$ & 0.007 & 0.005 & 0.08 & 0.00 & 0.006 & 2.70 & 18.75 & $0 \%$ & 1706 & 0.010 & 0.008 & 0.06 & 0.00 & 0.008 & 2.29 & 11.70 & $0 \%$ & 1436 \\
\hline \multicolumn{20}{|l|}{ Chile } \\
\hline \multirow[t]{2}{*}{ Mercado total } & $R$ & 0.000 & 0.001 & 0.05 & -0.05 & 0.008 & -0.71 & 9.34 & $0 \%$ & 1692 & 0.000 & 0.001 & 0.10 & -0.07 & 0.009 & 0.50 & 17.25 & $0 \%$ & 1425 \\
\hline & $C S A D$ & 0.010 & 0.009 & 0.05 & 0.00 & 0.004 & 2.31 & 17.15 & $0 \%$ & 1692 & 0.010 & 0.009 & 0.11 & 0.00 & 0.004 & 10.70 & 243.9 & $0 \%$ & 1425 \\
\hline \multirow[t]{2}{*}{ Alimentos y bebidas } & $R$ & 0.000 & 0.000 & 0.06 & -0.06 & 0.011 & -0.33 & 6.40 & $0 \%$ & 1677 & 0.000 & 0.000 & 0.08 & -0.05 & 0.009 & 0.34 & 8.05 & $0 \%$ & 1424 \\
\hline & $C S A D$ & 0.009 & 0.007 & 0.10 & 0.00 & 0.007 & 2.89 & 22.19 & $0 \%$ & 1677 & 0.008 & 0.008 & 0.04 & 0.00 & 0.005 & 1.51 & 7.49 & $0 \%$ & 1424 \\
\hline \multirow[t]{2}{*}{ Banca, financiero } & $R$ & 0.000 & 0.000 & 0.06 & -0.05 & 0.009 & -0.23 & 8.01 & $0 \%$ & 1687 & 0.001 & 0.001 & 0.10 & -0.07 & 0.011 & 0.34 & 11.21 & $0 \%$ & 1424 \\
\hline & $C S A D$ & 0.008 & 0.007 & 0.05 & 0.00 & 0.005 & 2.49 & 16.46 & $0 \%$ & 1687 & 0.008 & 0.007 & 0.04 & 0.00 & 0.004 & 1.62 & 7.97 & $0 \%$ & 1424 \\
\hline \multirow[t]{2}{*}{ Comercial, distribución } & $R$ & 0.000 & 0.001 & 0.06 & -0.07 & 0.013 & -0.11 & 5.36 & $0 \%$ & 1668 & 0.000 & 0.000 & 0.10 & -0.08 & 0.013 & 0.32 & 8.45 & $0 \%$ & 1424 \\
\hline & $C S A D$ & 0.007 & 0.006 & 0.06 & 0.00 & 0.006 & 1.84 & 9.48 & $0 \%$ & 1668 & 0.007 & 0.006 & 0.03 & 0.00 & 0.005 & 1.44 & 6.06 & $0 \%$ & 1424 \\
\hline \multirow[t]{2}{*}{ Inversión } & $R$ & 0.001 & 0.000 & 0.08 & -0.08 & 0.010 & -0.29 & 11.70 & $0 \%$ & 1589 & 0.000 & 0.000 & 0.07 & -0.08 & 0.010 & 0.01 & 10.14 & $0 \%$ & 1423 \\
\hline & $C S A D$ & 0.009 & 0.007 & 0.08 & 0.00 & 0.007 & 3.11 & 23.30 & $0 \%$ & 1589 & 0.009 & 0.009 & 0.04 & 0.00 & 0.005 & 1.40 & 6.82 & $0 \%$ & 1423 \\
\hline \multirow[t]{2}{*}{ Servicios públicos } & $R$ & 0.000 & 0.001 & 0.07 & -0.09 & 0.011 & -0.53 & 9.60 & $0 \%$ & 1692 & 0.000 & 0.000 & 0.12 & -0.05 & 0.009 & 1.52 & 28.88 & $0 \%$ & 1425 \\
\hline & $C S A D$ & 0.010 & 0.009 & 0.15 & 0.00 & 0.007 & 8.21 & 147.3 & $0 \%$ & 1692 & 0.008 & 0.007 & 0.03 & 0.00 & 0.003 & 1.31 & 6.22 & $0 \%$ & 1425 \\
\hline \multirow[t]{2}{*}{ Servicios varios } & $R$ & 0.000 & 0.000 & 0.05 & -0.08 & 0.012 & -0.74 & 8.17 & $0 \%$ & 740 & 0.001 & 0.000 & 0.41 & -0.09 & 0.015 & 14.20 & 387.6 & $0 \%$ & 1424 \\
\hline & $C S A D$ & 0.009 & 0.007 & 0.05 & 0.00 & 0.006 & 1.88 & 9.75 & $0 \%$ & 740 & 0.009 & 0.008 & 0.68 & 0.00 & 0.019 & 32.75 & 1175 & $0 \%$ & 1424 \\
\hline \multirow[t]{2}{*}{ Demás empresas } & $R$ & 0.001 & 0.001 & 0.08 & -0.08 & 0.011 & -0.36 & 8.63 & $0 \%$ & 1680 & 0.000 & 0.000 & 0.09 & -0.09 & 0.013 & -0.04 & 9.01 & $0 \%$ & 1424 \\
\hline & $C S A D$ & 0.011 & 0.009 & 0.07 & 0.00 & 0.007 & 2.25 & 12.61 & $0 \%$ & 1680 & 0.011 & 0.010 & 0.08 & 0.00 & 0.007 & 3.08 & 23.43 & $0 \%$ & 1424 \\
\hline \multicolumn{20}{|l|}{ Colombia } \\
\hline \multirow[t]{2}{*}{ Mercado total } & $R$ & 0.001 & 0.002 & 0.16 & -0.12 & 0.015 & -0.27 & 18.48 & $0 \%$ & 1654 & 0.001 & 0.001 & 0.08 & -0.06 & 0.010 & -0.28 & 9.88 & $0 \%$ & 1393 \\
\hline & $C S A D$ & 0.011 & 0.010 & 0.05 & 0.00 & 0.005 & 1.63 & 7.98 & $0 \%$ & 1654 & 0.010 & 0.010 & 0.09 & 0.00 & 0.005 & 5.84 & 79.04 & $0 \%$ & 1393 \\
\hline \multirow[t]{2}{*}{ Financiero } & $R$ & 0.002 & 0.002 & 0.14 & -0.12 & 0.017 & -0.25 & 11.01 & $0 \%$ & 1640 & 0.001 & 0.001 & 0.09 & -0.07 & 0.010 & 0.21 & 13.27 & $0 \%$ & 1393 \\
\hline & $C S A D$ & 0.009 & 0.007 & 0.11 & 0.00 & 0.008 & 3.53 & 27.78 & $0 \%$ & 1640 & 0.008 & 0.007 & 0.03 & 0.00 & 0.004 & 1.20 & 4.94 & $0 \%$ & 1393 \\
\hline \multirow[t]{2}{*}{ Inversión } & $R$ & 0.001 & 0.001 & 0.18 & -0.13 & 0.017 & -0.06 & 18.69 & $0 \%$ & 1644 & 0.001 & 0.001 & 0.08 & -0.06 & 0.011 & 0.11 & 7.79 & $0 \%$ & 1393 \\
\hline & $C S A D$ & 0.008 & 0.007 & 0.06 & 0.00 & 0.006 & 2.17 & 10.74 & $0 \%$ & 1644 & 0.007 & 0.006 & 0.04 & 0.00 & 0.004 & 1.75 & 8.55 & $0 \%$ & 1393 \\
\hline Petróleo & $R$ & 0.000 & 0.000 & 0.06 & -0.21 & 0.017 & -1.77 & 23.24 & $0 \%$ & 1104 & 0.000 & 0.000 & 0.06 & -0.21 & 0.017 & -1.77 & 23.24 & $0 \%$ & 1104 \\
\hline & $C S A D$ & 0.011 & 0.009 & 0.20 & 0.00 & 0.011 & 6.92 & 106.6 & $0 \%$ & 1104 & 0.011 & 0.009 & 0.20 & 0.00 & 0.011 & 6.92 & 106.6 & $0 \%$ & 1104 \\
\hline Energía & $R$ & 0.001 & 0.001 & 0.19 & -0.15 & 0.019 & -0.11 & 16.27 & $0 \%$ & 1609 & 0.000 & 0.001 & 0.09 & -0.06 & 0.012 & 0.39 & 7.90 & $0 \%$ & 1390 \\
\hline & $C S A D$ & 0.008 & 0.005 & 0.09 & 0.00 & 0.008 & 2.98 & 18.37 & $0 \%$ & 1609 & 0.007 & 0.006 & 0.04 & 0.00 & 0.005 & 1.84 & 8.76 & $0 \%$ & 1390 \\
\hline
\end{tabular}


Tabla 1 (Continuación)

\begin{tabular}{|c|c|c|c|c|c|c|c|c|c|c|c|c|c|c|c|c|c|c|c|}
\hline \multirow[t]{2}{*}{ Sector } & & \multicolumn{9}{|c|}{ Precrisis } & \multicolumn{9}{|c|}{ Poscrisis } \\
\hline & & $\bar{x}$ & $\tilde{x}$ & Max & Min & $\sigma$ & $S$ & $C$ & $P_{j b}$ & $N$ & $\overline{\bar{x}}$ & $\tilde{x}$ & $\operatorname{Max}$ & Min & $\sigma$ & $S$ & $C$ & $P_{j b}$ & $N$ \\
\hline \multirow[t]{2}{*}{ Demás empresas } & $R$ & 0.000 & 0.000 & 0.14 & -0.12 & 0.018 & 0.02 & 11.29 & $0 \%$ & 1587 & 0.000 & 0.001 & 0.12 & -0.15 & 0.013 & -1.39 & 24.62 & $0 \%$ & 1393 \\
\hline & $C S A D$ & 0.012 & 0.010 & 0.08 & 0.00 & 0.010 & 2.01 & 8.98 & $0 \%$ & 1587 & 0.013 & 0.011 & 0.26 & 0.00 & 0.012 & 10.32 & 173.2 & $0 \%$ & 1393 \\
\hline \multicolumn{20}{|l|}{ Perú } \\
\hline \multirow[t]{2}{*}{ Mercado total } & $R$ & 0.001 & 0.001 & 0.07 & -0.08 & 0.013 & -0.65 & 8.64 & $0 \%$ & 1690 & 0.000 & 0.000 & 0.08 & -0.16 & 0.015 & -0.98 & 16.63 & $0 \%$ & 1433 \\
\hline & $C S A D$ & 0.015 & 0.013 & 0.09 & 0.00 & 0.009 & 2.03 & 10.83 & $0 \%$ & 1690 & 0.014 & 0.013 & 0.06 & 0.00 & 0.006 & 2.38 & 12.87 & $0 \%$ & 1433 \\
\hline \multirow[t]{2}{*}{ Minero } & $R$ & 0.002 & 0.002 & 0.15 & -0.16 & 0.026 & -0.42 & 11.34 & $0 \%$ & 1558 & 0.000 & 0.001 & 0.11 & -0.16 & 0.020 & -0.67 & 11.93 & $0 \%$ & 1433 \\
\hline & $C S A D$ & 0.018 & 0.013 & 0.19 & 0.00 & 0.019 & 4.47 & 29.55 & $0 \%$ & 1558 & 0.017 & 0.015 & 0.07 & 0.00 & 0.009 & 1.58 & 7.24 & $0 \%$ & 1433 \\
\hline \multirow[t]{2}{*}{ Servicios públicos } & $R$ & 0.001 & 0.001 & 0.06 & -0.09 & 0.012 & -0.56 & 8.38 & $0 \%$ & 1351 & 0.001 & 0.001 & 0.12 & -0.14 & 0.013 & -0.68 & 28.53 & $0 \%$ & 1245 \\
\hline & $C S A D$ & 0.008 & 0.007 & 0.08 & 0.00 & 0.007 & 2.47 & 15.76 & $0 \%$ & 1351 & 0.007 & 0.006 & 0.06 & 0.00 & 0.005 & 1.91 & 10.80 & $0 \%$ & 1245 \\
\hline \multirow[t]{2}{*}{ Industrial } & $R$ & 0.001 & 0.001 & 0.08 & -0.09 & 0.016 & -0.20 & 6.04 & $0 \%$ & 1586 & 0.000 & 0.000 & 0.11 & -0.16 & 0.018 & -0.48 & 13.52 & $0 \%$ & 1433 \\
\hline & $C S A D$ & 0.016 & 0.013 & 0.08 & 0.00 & 0.011 & 1.55 & 5.93 & $0 \%$ & 1586 & 0.014 & 0.012 & 0.13 & 0.00 & 0.009 & 3.39 & 33.68 & $0 \%$ & 1433 \\
\hline \multirow[t]{2}{*}{ Diversas } & $R$ & 0.002 & 0.003 & 0.14 & -0.14 & 0.025 & -0.15 & 8.98 & $0 \%$ & 1136 & 0.001 & 0.001 & 0.10 & -0.16 & 0.014 & -0.84 & 18.23 & $0 \%$ & 1396 \\
\hline & $C S A D$ & 0.017 & 0.012 & 0.14 & 0.00 & 0.018 & 2.99 & 14.99 & $0 \%$ & 1136 & 0.009 & 0.008 & 0.10 & 0.00 & 0.007 & 3.49 & 32.93 & $0 \%$ & 1396 \\
\hline \multicolumn{20}{|l|}{ Argentina } \\
\hline \multirow[t]{2}{*}{ Mercado total } & $R$ & 0.001 & 0.002 & 0.16 & -0.10 & 0.020 & 0.45 & 10.38 & $0 \%$ & 1676 & 0.001 & 0.002 & 0.11 & -0.13 & 0.020 & -0.70 & 8.10 & $0 \%$ & 1388 \\
\hline & $C S A D$ & 0.017 & 0.014 & 0.16 & 0.00 & 0.013 & 4.76 & 39.57 & $0 \%$ & 1676 & 0.016 & 0.014 & 0.08 & 0.00 & 0.007 & 2.46 & 14.74 & $0 \%$ & 1388 \\
\hline \multirow[t]{2}{*}{ Siderurgia } & $R$ & 0.001 & 0.001 & 0.09 & -0.11 & 0.017 & -0.27 & 5.92 & $0 \%$ & 1602 & 0.001 & 0.001 & 0.11 & -0.16 & 0.022 & -0.61 & 8.20 & $0 \%$ & 1388 \\
\hline & $C S A D$ & 0.011 & 0.010 & 0.07 & 0.00 & 0.008 & 1.68 & 8.10 & $0 \%$ & 1602 & 0.011 & 0.010 & 0.08 & 0.00 & 0.008 & 2.23 & 11.82 & $0 \%$ & 1388 \\
\hline \multirow[t]{2}{*}{ Petróleo, gas } & $R$ & 0.001 & 0.001 & 0.20 & -0.10 & 0.022 & 0.88 & 10.86 & $0 \%$ & 1122 & 0.001 & 0.001 & 0.15 & -0.15 & 0.022 & -0.47 & 8.48 & $0 \%$ & 1359 \\
\hline & $C S A D$ & 0.014 & 0.011 & 0.09 & 0.00 & 0.013 & 1.72 & 7.15 & $0 \%$ & 1122 & 0.013 & 0.010 & 0.16 & 0.00 & 0.012 & 3.54 & 26.95 & $0 \%$ & 1359 \\
\hline \multirow[t]{2}{*}{ Bancos } & $R$ & 0.001 & 0.001 & 0.38 & -0.16 & 0.029 & 1.33 & 24.63 & $0 \%$ & 1673 & 0.002 & 0.001 & 0.15 & -0.18 & 0.027 & -0.17 & 7.00 & $0 \%$ & 1388 \\
\hline & $C S A D$ & 0.011 & 0.008 & 0.12 & 0.00 & 0.011 & 4.02 & 27.86 & $0 \%$ & 1673 & 0.010 & 0.008 & 0.06 & 0.00 & 0.007 & 1.94 & 10.13 & $0 \%$ & 1388 \\
\hline \multirow[t]{2}{*}{ Demás empresas } & $R$ & 0.001 & 0.000 & 0.29 & -0.27 & 0.032 & 0.76 & 18.82 & $0 \%$ & 1667 & 0.001 & 0.001 & 0.10 & -0.19 & 0.023 & -0.62 & 8.84 & $0 \%$ & 1388 \\
\hline & $C S A D$ & 0.017 & 0.012 & 0.39 & 0.00 & 0.023 & 6.86 & 80.71 & $0 \%$ & 1667 & 0.015 & 0.013 & 0.10 & 0.00 & 0.011 & 2.64 & 15.10 & $0 \%$ & 1388 \\
\hline
\end{tabular}

Simbología: $\bar{x}$ media, $\tilde{x}$ mediana, Max máximo, Min mínimo, $\sigma$ desviación estándar, $S$ sesgo, $C$ curtosis, $P_{j b}$ probabilidad estadístico Jarque-Bera, $N$ número de datos.

Abreviaturas: Construcción (Constr.), Telecomunicaciones (Telec.), Petroquímico (PQ), Alimentos, Bebidas y Tabaco (A.B.T.).

Variables: $R$ Rentabilidad logarítmica, CSAD Desviación absoluta de sección cruzada.

Fuente: elaboración propia. 


\section{Modelo I precrisis}

En la tabla 2 se presenta la estimación del modelo I en los sectores de Latinoamérica para el subperiodo precrisis.

Teniendo en cuenta los resultados presentados en la tabla 1 , se puede observar que, a excepción de algunos coeficientes en el mercado bursátil de Brasil ( $\beta 1$ en los criterios $1 \%$ y $10 \%$ del sector de siderurgia; $\beta 2$ del sector de madera y papel en el criterio $5 \%$; $\beta 2$ en el sector de minería en el criterio $1 \%$, y $\beta 1$ en el sector bancario en el criterio $10 \%$ ), los coeficientes $\beta_{1}$ y $\beta_{2}$ son positivos y estadísticamente significativos tanto para el total del mercado como para los sectores que lo componen, en los países objeto de estudio; esto sugiere que la dispersión (CSAD) se incrementa durante periodos de extremo movimiento del mercado, lo cual indica ausencia de efecto manada.

También se puede apreciar que los sectores que exhiben los menores valores de dispersión promedio durante días de normalidad en el precio de los activos (denotados por $\alpha$ ) son: en el mercado de Brasil el sector minero; en el mercado de México el sector de demás empresas; en el mercado de Chile el sector de comerciales y distribuidoras; en el mercado de Colombia el sector de energía; en el mercado de Perú el sector de servicios públicos, y en el mercado de Argentina el sector bancario.

Además, de manera general, considerando los 3 criterios seleccionados como extremos de la distribución de rentabilidad del mercado, se resalta que en las observaciones de caída en los precios de las acciones los niveles de dispersión son más bajos, lo que puede significar que las decisiones de los inversores son más uniformes cuando se presentan malas noticias.

\section{Modelo I poscrisis}

En la tabla 3 se presenta la estimación del modelo I en los sectores de Latinoamérica para el subperiodo poscrisis.

Examinando los resultados presentados en la tabla 2, se observa que todos los coeficientes son positivos y estadísticamente significativos durante los 3 criterios seleccionados como extremos, exceptuando, en el criterio $1 \%$, el coeficiente $\beta 2$ en el sector de madera y papel del mercado de Brasil y los coeficientes $\beta 1$ y $\beta 2$ del mercado de chile para los sectores de comerciales y distribuidoras y servicios varios, respectivamente, lo que significa que en promedio la dispersión aumenta durante cambios bruscos en el precio de las acciones y, por tanto, no está presente el efecto manada.

Los menores valores de dispersión promedio en días de movimientos regulares en el mercado los exhibe el sector bancario en Brasil; el sector de demás empresas en México; el sector de comerciales y distribuidoras en Chile; el sector de inversiones en Colombia; el sector de servicios públicos en Perú, y el sector bancario en Argentina.

\section{Modelo I periodo total}

Los resultados del modelo I para el periodo total (2002-2014) mostraron ausencia de efecto manada, teniendo en cuenta que los coeficientes $\beta 1$ y $\beta 2$ de las plazas bursátiles objeto de estudio, tanto del total del mercado como de los sectores al interior de este, se presentaron positivos y estadísticamente significativos. No se reporta la respectiva tabla debido a que los resultados son similares a los de las tablas 1 y 2 de pre y poscrisis presentadas anteriormente.

\section{Modelo II}

En esta sección se presentan los resultados de la regresión no lineal propuesta por Chang et al. (2000), tanto para la muestra total como para las submuestras de rentabilidades mayores y 
Tabla 2

Modelo I en Latinoamérica por sectores periodo precrisis

\begin{tabular}{|c|c|c|c|c|c|c|c|c|c|}
\hline \multirow[t]{2}{*}{ Sector } & \multicolumn{3}{|c|}{ Criterio $1 \%$} & \multicolumn{3}{|c|}{ Criterio 5\% } & \multicolumn{3}{|c|}{ Criterio $10 \%$} \\
\hline & $\alpha$ & $\beta 1$ & $\beta 2$ & $\alpha$ & $\beta 1$ & $\beta 2$ & $\alpha$ & $\beta 1$ & $\beta 2$ \\
\hline \multicolumn{10}{|l|}{ Brasil } \\
\hline Mercado total & $0.0162 *$ & $0.0105^{*}$ & $0.0118 *$ & $0.0158 *$ & $0.0058^{*}$ & $0.0065^{*}$ & $0.0156^{*}$ & $0.0041 *$ & $0.0043 *$ \\
\hline Bancario & $0.0090^{*}$ & $0.0053^{*}$ & $0.0034 * *$ & $0.0090 *$ & $0.0014 * *$ & $0.0018 * *$ & $0.0089^{*}$ & 0.0005 & $0.0020 *$ \\
\hline Otros financieros & $0.0118^{*}$ & $0.0180 *$ & $0.0161 *$ & $0.0113^{*}$ & $0.0087^{*}$ & $0.0083^{*}$ & $0.0108^{*}$ & $0.0062 *$ & $0.0074 *$ \\
\hline Construcción civil & $0.0176^{*}$ & $0.0222 * *$ & $0.0306^{*}$ & $0.0171 *$ & $0.0070^{* * *}$ & $0.0144 *$ & $0.0168^{*}$ & $0.0042 * *$ & $0.0090 *$ \\
\hline Servicios públicos & $0.0144^{*}$ & $0.0076^{*}$ & $0.0128 *$ & $0.0140^{*}$ & $0.0064 *$ & $0.0059^{*}$ & $0.0138^{*}$ & $0.0037 *$ & $0.0042 *$ \\
\hline Consumo cíclico & $0.0184^{*}$ & $0.1515^{*}$ & $0.1929 *$ & $0.0162 *$ & $0.0520^{*}$ & $0.0639 *$ & $0.0153^{*}$ & $0.0286^{*}$ & $0.0375^{*}$ \\
\hline Consumo no cíclico & $0.0143^{*}$ & $0.0059 *$ & $0.0089 * *$ & $0.0139 *$ & $0.0055^{*}$ & $0.0052 *$ & $0.0136^{*}$ & $0.0038 *$ & $0.0048 *$ \\
\hline Madera y papel & $0.0113^{*}$ & $0.0289 * *$ & $0.0294 *$ & $0.0109 *$ & $0.0157 *$ & 0.0061 & $0.0108 *$ & $0.0065^{* *}$ & $0.0055^{* *}$ \\
\hline Siderurgia & $0.0104 *$ & 0.0028 & $0.0035^{* * *}$ & $0.0102 *$ & $0.0021 * * *$ & $0.0028^{*}$ & $0.0101 *$ & 0.0011 & $0.0023 *$ \\
\hline Transporte & $0.0149 *$ & $0.0312 *$ & $0.0623 * *$ & $0.0144 *$ & $0.0116^{*}$ & $0.0180 * *$ & $0.0139 *$ & $0.0074 *$ & $0.0117 *$ \\
\hline Minero & $0.0058 *$ & $0.0170 * *$ & 0.0044 & $0.0055^{*}$ & $0.0064 * *$ & $0.0037 * *$ & $0.0053 *$ & $0.0043 *$ & $0.0028 * *$ \\
\hline Telecomunicaciones & $0.0128 *$ & $0.0126^{*}$ & $0.0152 *$ & $0.0125^{*}$ & $0.0047 *$ & $0.0072 *$ & $0.0123^{*}$ & $0.0031 *$ & $0.0051 *$ \\
\hline Petroquímicos & $0.0102 *$ & $0.0065^{* *}$ & $0.0155^{*}$ & $0.0100 *$ & $0.0022 * *$ & $0.0076^{*}$ & $0.0096^{*}$ & $0.0021 *$ & $0.0061 *$ \\
\hline \multicolumn{10}{|l|}{ México } \\
\hline Mercado total & $0.0125^{*}$ & $0.0140^{*}$ & $0.0097 *$ & $0.0121 *$ & $0.0074 *$ & $0.0063^{*}$ & $0.0118^{*}$ & $0.0050^{*}$ & $0.0049 *$ \\
\hline Bienes de consumo NB & $0.0098^{*}$ & $0.0181 *$ & $0.0195^{*}$ & $0.0090 *$ & $0.0102 *$ & $0.0135^{*}$ & $0.0084^{*}$ & $0.0078^{*}$ & $0.0106^{*}$ \\
\hline Materiales & $0.0132 *$ & $0.0246^{*}$ & $0.0270^{*}$ & $0.0124 *$ & $0.0138 *$ & $0.0129^{*}$ & $0.0118^{*}$ & $0.0089^{*}$ & $0.0109 *$ \\
\hline Alimentos, bebidas y tabaco & $0.0099^{*}$ & $0.0071^{*}$ & $0.0087 *$ & $0.0096^{*}$ & $0.0051 *$ & $0.0046^{*}$ & $0.0092 *$ & $0.0042 *$ & $0.0042 *$ \\
\hline Entidades financieras & $0.0101 *$ & $0.0397 *$ & $0.0405^{*}$ & $0.0092 *$ & $0.0150^{*}$ & $0.0189 *$ & $0.0088^{*}$ & $0.0086^{*}$ & $0.0123 *$ \\
\hline Productos de consumo frec & $0.0087 *$ & $0.0270 * * *$ & $0.0083^{*}$ & $0.0083 *$ & $0.0090 * *$ & $0.0072 *$ & $0.0080^{*}$ & $0.0054 * *$ & $0.0058 *$ \\
\hline Industrial & $0.0118^{*}$ & $0.0215^{*}$ & $0.0342 *$ & $0.0110^{*}$ & $0.0104 *$ & $0.0156^{*}$ & $0.0104^{*}$ & $0.0082 *$ & $0.0114^{*}$ \\
\hline Demás empresas & $0.0068^{*}$ & $0.0124 *$ & $0.0083 *$ & $0.0065^{*}$ & $0.0056^{*}$ & $0.0051^{*}$ & $0.0063^{*}$ & $0.0034^{*}$ & $0.0036^{*}$ \\
\hline \multicolumn{10}{|l|}{ Chile } \\
\hline Mercado total & $0.0097 *$ & $0.0114 *$ & $0.0111 *$ & $0.0094 *$ & $0.0053^{*}$ & $0.0062 *$ & $0.0091 *$ & $0.0038 *$ & $0.0047 *$ \\
\hline Alimentos y bebidas & $0.0088^{*}$ & $0.0160 *$ & $0.0134 *$ & $0.0081 *$ & $0.0101 *$ & $0.0100 *$ & $0.0075^{*}$ & $0.0076^{*}$ & $0.0085^{*}$ \\
\hline Bancarias y financieras & $0.0074^{*}$ & $0.0133^{*}$ & $0.0089 *$ & $0.0070^{*}$ & $0.0067 *$ & $0.0067^{*}$ & $0.0067^{*}$ & $0.0047^{*}$ & $0.0050 *$ \\
\hline Comerciales y distribuidoras & $0.0069 *$ & $0.0056^{*}$ & $0.0080 * *$ & $0.0066^{*}$ & $0.0028 *$ & $0.0068^{*}$ & $0.0063^{*}$ & $0.0020^{*}$ & $0.0051 *$ \\
\hline Inversiones e inmobiliarias & $0.0084 *$ & $0.0142 *$ & $0.0257 *$ & $0.0076^{*}$ & $0.0095 *$ & $0.0128 *$ & $0.0071 *$ & $0.0069 *$ & $0.0097 *$ \\
\hline Servicios públicos & $0.0096^{*}$ & $0.0164 * * *$ & $0.0162 *$ & $0.0092 *$ & $0.0066^{*}$ & $0.0076^{*}$ & $0.0089 *$ & $0.0050 *$ & $0.0059 *$ \\
\hline
\end{tabular}


Tabla 2 (continuación)

\begin{tabular}{|c|c|c|c|c|c|c|c|c|c|}
\hline \multirow[t]{2}{*}{ Sector } & \multicolumn{3}{|c|}{ Criterio $1 \%$} & \multicolumn{3}{|c|}{ Criterio 5\% } & \multicolumn{3}{|c|}{ Criterio $10 \%$} \\
\hline & $\alpha$ & $\beta 1$ & $\beta 2$ & $\alpha$ & $\beta 1$ & $\beta 2$ & $\alpha$ & $\beta 1$ & $\beta 2$ \\
\hline Servicios varios & $0.0083^{*}$ & $0.0107 *$ & $0.0137 *$ & $0.0080 *$ & $0.0057 *$ & $0.0061 *$ & $0.0075^{*}$ & $0.0045^{*}$ & $0.0062 *$ \\
\hline Demás empresas & $0.0102 *$ & $0.0156^{*}$ & $0.0238^{*}$ & $0.0095 *$ & $0.0089 *$ & $0.0132 *$ & $0.0090 *$ & $0.0067^{*}$ & $0.0097^{*}$ \\
\hline \multicolumn{10}{|l|}{ Colombia } \\
\hline Mercado total & $0.0108 *$ & $0.0110^{*}$ & $0.0121 *$ & $0.0102 *$ & $0.0063 *$ & $0.0094^{*}$ & $0.0097 *$ & $0.0051 *$ & $0.0078 *$ \\
\hline Financiero & $0.0090 *$ & $0.0136^{* *}$ & $0.0196^{*}$ & $0.0081 *$ & $0.0099 *$ & $0.0152 *$ & $0.0076^{*}$ & $0.0070^{*}$ & $0.0109 *$ \\
\hline Inversiones & $0.0082 *$ & $0.0123 *$ & $0.0106^{*}$ & $0.0075^{*}$ & $0.0078 *$ & $0.0101 *$ & $0.0070^{*}$ & $0.0056^{*}$ & $0.0086^{*}$ \\
\hline Energía & $0.0073 *$ & $0.0181 *$ & $0.0141 *$ & $0.0066^{*}$ & $0.0098 *$ & $0.0114 *$ & $0.0061 *$ & $0.0063^{*}$ & $0.0089 *$ \\
\hline Demás empresas & $0.0121 *$ & $0.0121 *$ & $0.0153^{*}$ & $0.0111^{*}$ & $0.0093 *$ & $0.0175^{*}$ & $0.0101 *$ & $0.0084^{*}$ & $0.0144^{*}$ \\
\hline \multicolumn{10}{|l|}{ Perú } \\
\hline Mercado total & $0.0150 *$ & $0.0206^{*}$ & $0.0264 *$ & $0.0139 *$ & $0.0141 *$ & $0.0172 *$ & $0.0132 *$ & $0.0102 *$ & $0.0124 *$ \\
\hline Mineras & $0.0160 *$ & $0.0988^{*}$ & $0.0773 *$ & $0.0143 *$ & $0.0352 *$ & $0.0351 *$ & $0.0132 *$ & $0.0218 *$ & $0.0244 *$ \\
\hline Servicios públicos & $0.0079 *$ & $0.0228 *$ & $0.0154 *$ & $0.0072 *$ & $0.0107 *$ & $0.0108 *$ & $0.0066^{*}$ & $0.0084 *$ & $0.0079 *$ \\
\hline Industrial & $0.0153 *$ & $0.0179 *$ & $0.0276^{*}$ & $0.0141 *$ & $0.0145^{*}$ & $0.0196^{*}$ & $0.0131 *$ & $0.0103^{*}$ & $0.0172 *$ \\
\hline Diversas & $0.0160 *$ & $0.0530 *$ & $0.0470 *$ & $0.0134 *$ & $0.0336^{*}$ & $0.0384^{*}$ & $0.0122 *$ & $0.0226^{*}$ & $0.0264^{*}$ \\
\hline \multicolumn{10}{|l|}{ Argentina } \\
\hline Mercado total & $0.0165^{*}$ & $0.0238^{*}$ & $0.0540 *$ & $0.0155^{*}$ & $0.0140 *$ & $0.0224 *$ & $0.0150 *$ & $0.0090^{*}$ & $0.0138 *$ \\
\hline Metalúrgica y siderúrgica & $0.0107 *$ & $0.0121 *$ & $0.0160 *$ & $0.0104 *$ & $0.0040 *$ & $0.0089 *$ & $0.0100^{*}$ & $0.0029 *$ & $0.0076^{*}$ \\
\hline Petróleo y gas & $0.0139 *$ & $0.0306^{*}$ & $0.0174 * *$ & $0.0128 *$ & $0.0172 *$ & $0.0140 *$ & $0.0119 *$ & $0.0133^{*}$ & $0.0118^{*}$ \\
\hline Bancos & $0.0104 *$ & $0.0152 * *$ & $0.0282 *$ & $0.0097 *$ & $0.0098 *$ & $0.0134 *$ & $0.0093^{*}$ & $0.0061 *$ & $0.0094 *$ \\
\hline \multirow[t]{2}{*}{ Demás empresas } & $0.0159 *$ & $0.0645^{*}$ & $0.0894 *$ & $0.0143 *$ & $0.0266^{*}$ & $0.0363^{*}$ & $0.0130 *$ & $0.0180^{*}$ & $0.0263^{*}$ \\
\hline & $0.000 \%$ & $0.504 \%$ & $0.024 \%$ & $0.000 \%$ & $0.002 \%$ & $0.000 \%$ & $0.000 \%$ & $0.000 \%$ & $0.000 \%$ \\
\hline
\end{tabular}

El estadístico-t basado en Newey y West (1987) consistente con heterocedasticidad y autocorrelación es usado para probar la hipótesis.

$*$, ** y ***: valores significativos al $1 \%$, al $5 \%$ y al $10 \%$, respectivamente.

Fuente: elaboración propia. 
Modelo I en Latinoamérica por sectores periodo poscrisis

\begin{tabular}{|c|c|c|c|c|c|c|c|c|c|}
\hline \multirow[t]{2}{*}{ Sector } & \multicolumn{3}{|c|}{ Criterio $1 \%$} & \multicolumn{3}{|c|}{ Criterio 5\% } & \multicolumn{3}{|c|}{ Criterio $10 \%$} \\
\hline & $\alpha$ & $\beta 1$ & $\beta 2$ & $\alpha$ & $\beta 1$ & $\beta 2$ & $\alpha$ & $\beta 1$ & $\beta 2$ \\
\hline \multicolumn{10}{|l|}{ Brasil } \\
\hline Mercado total & $0.0145^{*}$ & $0.0181 *$ & $0.0189 *$ & $0.0141 *$ & $0.0080^{*}$ & $0.0088^{*}$ & $0.0138^{*}$ & $0.0051 *$ & $0.0064 *$ \\
\hline Bancario & $0.0068 *$ & $0.0068 *$ & $0.0142 *$ & $0.0066^{*}$ & $0.0036^{*}$ & $0.0055^{*}$ & $0.0065^{*}$ & $0.0022 *$ & $0.0034 *$ \\
\hline Otros financieros & $0.0122 *$ & $0.0203 *$ & $0.0203^{*}$ & $0.0117 *$ & $0.0085^{*}$ & $0.0087 *$ & $0.0113^{*}$ & $0.0065^{*}$ & $0.0060^{*}$ \\
\hline Construcción civil & $0.0144 *$ & $0.0212 *$ & $0.0181^{*}$ & $0.0140^{*}$ & $0.0068 *$ & $0.0091 *$ & $0.0137^{*}$ & $0.0046^{*}$ & $0.0061 *$ \\
\hline Servicios públicos & $0.0114 *$ & $0.0141 *$ & $0.0122 *$ & $0.0110^{*}$ & $0.0085^{*}$ & $0.0055^{*}$ & $0.0106^{*}$ & $0.0056^{*}$ & $0.0047 *$ \\
\hline Consumo cíclico & $0.0129 *$ & $0.0192 *$ & $0.0199 *$ & $0.0124 *$ & $0.0081 *$ & $0.0095^{*}$ & $0.0121^{*}$ & $0.0049 *$ & $0.0068^{*}$ \\
\hline Consumo no cíclico & $0.0137 *$ & $0.0139 *$ & $0.0178^{*}$ & $0.0133 *$ & $0.0068 *$ & $0.0085^{*}$ & $0.0130 *$ & $0.0047 *$ & $0.0062 *$ \\
\hline Madera y papel & $0.0111^{*}$ & $0.0050 * * *$ & 0.0169 & $0.0108 *$ & $0.0031 *$ & $0.0079 *$ & $0.0107 *$ & $0.0014 * * *$ & $0.0052^{*}$ \\
\hline Siderurgia & $0.0088^{*}$ & $0.0059^{*}$ & $0.0128 *$ & $0.0085^{*}$ & $0.0034^{*}$ & $0.0069 *$ & $0.0083^{*}$ & $0.0024^{*}$ & $0.0046^{*}$ \\
\hline Transporte & $0.0139 *$ & $0.0160 *$ & $0.0278^{*}$ & $0.0134 *$ & $0.0090 *$ & $0.0118^{*}$ & $0.0131 *$ & $0.0053 *$ & $0.0073 *$ \\
\hline Minero & $0.0123 *$ & $0.0283 *$ & $0.1064 * *$ & $0.0112 *$ & $0.0166^{*}$ & $0.0353 *$ & $0.0106^{*}$ & $0.0106^{*}$ & $0.0208 *$ \\
\hline Telecomunicaciones & $0.0115^{*}$ & $0.0334 *$ & $0.0293^{*}$ & $0.0109 *$ & $0.0144 *$ & $0.0121 *$ & $0.0104 *$ & $0.0102 *$ & $0.0076^{*}$ \\
\hline Petroquímicos & $0.0088^{*}$ & $0.0128 *$ & $0.0187 *$ & $0.0085^{*}$ & $0.0048^{*}$ & $0.0084 *$ & $0.0082^{*}$ & $0.0030^{*}$ & $0.0065^{*}$ \\
\hline \multicolumn{10}{|l|}{ México } \\
\hline Mercado total & $0.0123 *$ & $0.0163 *$ & $0.0228^{*}$ & $0.0118^{*}$ & $0.0081^{*}$ & $0.0112 *$ & $0.0114 *$ & $0.0056^{*}$ & $0.0078^{*}$ \\
\hline Bienes de consumo NB & $0.0106^{*}$ & $0.0228 *$ & $0.0276^{*}$ & $0.0098 *$ & $0.0132 *$ & $0.0131 *$ & $0.0091^{*}$ & $0.0101 *$ & $0.0106^{*}$ \\
\hline Materiales & $0.0123 *$ & $0.0149 *$ & $0.0232 *$ & $0.0115^{*}$ & $0.0115^{*}$ & $0.0127 *$ & $0.0112 *$ & $0.0069 *$ & $0.0079 *$ \\
\hline Alimentos, bebidas y tabaco & $0.0103 *$ & $0.0364 * *$ & $0.0278^{*}$ & $0.0097 *$ & $0.0111^{*}$ & $0.0130 *$ & $0.0094 *$ & $0.0061^{*}$ & $0.0092 *$ \\
\hline Entidades financieras & $0.0119 *$ & $0.0162 *$ & $0.0374 *$ & $0.0113 *$ & $0.0084 *$ & $0.0145^{*}$ & $0.0108^{*}$ & $0.0062 *$ & $0.0100 *$ \\
\hline Productos de consumo frec & $0.0098 *$ & $0.0120 *$ & $0.0426^{*}$ & $0.0092 *$ & $0.0076^{*}$ & $0.0160 *$ & $0.0089 *$ & $0.0045^{*}$ & $0.0104 *$ \\
\hline Industrial & $0.0113 *$ & $0.0097 *$ & $0.0157 *$ & $0.0107 *$ & $0.0077 *$ & $0.0095 *$ & $0.0103^{*}$ & $0.0054 *$ & $0.0068^{*}$ \\
\hline Demás empresas & $0.0096^{*}$ & $0.0103 *$ & $0.0182 *$ & $0.0090 *$ & $0.0070^{*}$ & $0.0112 *$ & $0.0086^{*}$ & $0.0055^{*}$ & $0.0070 *$ \\
\hline \multicolumn{10}{|l|}{ Chile } \\
\hline Mercado total & $0.0096^{*}$ & $0.0067 *$ & $0.0159 *$ & $0.0093 *$ & $0.0033^{*}$ & $0.0075^{*}$ & $0.0090 *$ & $0.0025^{*}$ & $0.0053^{*}$ \\
\hline Alimentos y bebidas & $0.0082 *$ & $0.0088 *$ & $0.0078^{*}$ & $0.0078 *$ & $0.0058 *$ & $0.0063 *$ & $0.0075^{*}$ & $0.0042 *$ & $0.0053^{*}$ \\
\hline Bancarias y financieras & $0.0080 *$ & $0.0080 *$ & $0.0084 *$ & $0.0078 *$ & $0.0027 *$ & $0.0056^{*}$ & $0.0076^{*}$ & $0.0019 *$ & $0.0044 *$ \\
\hline Comerciales y distribuidoras & $0.0073 *$ & 0.0007 & $0.0046^{*}$ & $0.0071 *$ & $0.0012 * *$ & $0.0046^{*}$ & $0.0069 *$ & $0.0013 *$ & $0.0040^{*}$ \\
\hline Inversiones e inmobiliarias & $0.0092 *$ & $0.0076^{*}$ & $0.0126^{*}$ & $0.0089 *$ & $0.0043^{*}$ & $0.0073 *$ & $0.0085^{*}$ & $0.0035^{*}$ & $0.0059 *$ \\
\hline Servicios públicos & $0.0078 *$ & $0.0050 *$ & $0.0068 *$ & $0.0076^{*}$ & $0.0030 *$ & $0.0045^{*}$ & $0.0074^{*}$ & $0.0020 *$ & $0.0037 *$ \\
\hline
\end{tabular}


Tabla 3 (continuación)

\begin{tabular}{|c|c|c|c|c|c|c|c|c|c|}
\hline \multirow[t]{2}{*}{ Sector } & \multicolumn{3}{|c|}{ Criterio $1 \%$} & \multicolumn{3}{|c|}{ Criterio 5\% } & \multicolumn{3}{|c|}{ Criterio $10 \%$} \\
\hline & $\alpha$ & $\beta 1$ & $\beta 2$ & $\alpha$ & $\beta 1$ & $\beta 2$ & $\alpha$ & $\beta 1$ & $\beta 2$ \\
\hline Servicios varios & $0.0087 *$ & $0.0091 *$ & 0.0625 & $0.0083 *$ & $0.0039 *$ & $0.0179 * * *$ & $0.0080 *$ & $0.0031 *$ & $0.0112 * *$ \\
\hline Demás empresas & $0.0108 *$ & $0.0133^{*}$ & $0.0216^{*}$ & $0.0103 *$ & $0.0066^{*}$ & $0.0115^{*}$ & $0.0099 *$ & $0.0047 *$ & $0.0083^{*}$ \\
\hline \multicolumn{10}{|l|}{ Colombia } \\
\hline Mercado total & $0.0100^{*}$ & $0.0126^{* *}$ & $0.0112 *$ & $0.0096^{*}$ & $0.0068^{*}$ & $0.0058^{*}$ & $0.0094 *$ & $0.0041^{*}$ & $0.0045^{*}$ \\
\hline Financiero & $0.0077 *$ & $0.0059^{*}$ & $0.0077 *$ & $0.0074 *$ & $0.0038 *$ & $0.0056^{*}$ & $0.0071^{*}$ & $0.0034 *$ & $0.0043^{*}$ \\
\hline Inversiones & $0.0071 *$ & $0.0076^{*}$ & $0.0067 *$ & $0.0068^{*}$ & $0.0041 *$ & $0.0053^{*}$ & $0.0064 *$ & $0.0038^{*}$ & $0.0044^{*}$ \\
\hline Petróleo & $0.0106^{*}$ & $0.0355^{* *}$ & $0.0230^{*}$ & $0.0100 *$ & $0.0140^{*}$ & $0.0098^{*}$ & $0.0097 *$ & $0.0086^{*}$ & $0.0065^{*}$ \\
\hline Energía & $0.0072 *$ & $0.0068 *$ & $0.0076^{* *}$ & $0.0069 *$ & $0.0046^{*}$ & $0.0046^{*}$ & $0.0065^{*}$ & $0.0041 *$ & $0.0045^{*}$ \\
\hline Demás empresas & $0.0118^{*}$ & $0.0539 *$ & $0.0320 *$ & $0.0111^{*}$ & $0.0167 *$ & $0.0151^{*}$ & $0.0105^{*}$ & $0.0103 *$ & $0.0109 *$ \\
\hline \multicolumn{10}{|l|}{ Perú } \\
\hline Mercado total & $0.0137 *$ & $0.0203 *$ & $0.0188^{*}$ & $0.0131 *$ & $0.0102 *$ & $0.0109^{*}$ & $0.0126^{*}$ & $0.0078^{*}$ & $0.0078^{*}$ \\
\hline Mineras & $0.0163 *$ & $0.0205^{*}$ & $0.0144 *$ & $0.0156^{*}$ & $0.0104 *$ & $0.0106^{*}$ & $0.0150 *$ & $0.0077 *$ & $0.0086^{*}$ \\
\hline Servicios públicos & $0.0069^{*}$ & $0.0113^{*}$ & $0.0139 *$ & $0.0064 *$ & $0.0071^{*}$ & $0.0078^{*}$ & $0.0060 *$ & $0.0061 *$ & $0.0063^{*}$ \\
\hline Industrial & $0.0135^{*}$ & $0.0220^{*}$ & $0.0132 * *$ & $0.0129 *$ & $0.0091^{*}$ & $0.0100^{*}$ & $0.0121 *$ & $0.0080^{*}$ & $0.0088^{*}$ \\
\hline Diversas & $0.0087 *$ & $0.0118 * * *$ & $0.0157 *$ & $0.0081 *$ & $0.0085^{*}$ & $0.0092^{*}$ & $0.0076^{*}$ & $0.0064^{*}$ & $0.0076^{*}$ \\
\hline \multicolumn{10}{|l|}{ Argentina } \\
\hline Mercado total & $0.0153^{*}$ & $0.0185^{* *}$ & $0.0170^{*}$ & $0.0148 *$ & $0.0073^{*}$ & $0.0098^{*}$ & $0.0143 *$ & $0.0053^{*}$ & $0.0080^{*}$ \\
\hline Metalúrgica y Siderúrgica & $0.0111^{*}$ & $0.0102 *$ & $0.0112 * *$ & $0.0105^{*}$ & $0.0055^{*}$ & $0.0103^{*}$ & $0.0101 *$ & $0.0044^{*}$ & $0.0075^{*}$ \\
\hline Petróleo y gas & $0.0127 *$ & $0.0396^{*}$ & $0.0283^{*}$ & $0.0119^{*}$ & $0.0149^{*}$ & $0.0154^{*}$ & $0.0112 *$ & $0.0104 *$ & $0.0116^{*}$ \\
\hline Bancos & $0.0098 *$ & $0.0082 *$ & $0.0075^{*}$ & $0.0095^{*}$ & $0.0022 * * *$ & $0.0051^{*}$ & $0.0095^{*}$ & 0.0009 & $0.0033^{*}$ \\
\hline Demás empresas & $0.0148 *$ & $0.0201 * *$ & $0.0205^{*}$ & $0.0140 *$ & $0.0082 *$ & $0.0163^{*}$ & $0.0136^{*}$ & $0.0051 *$ & $0.0117 *$ \\
\hline
\end{tabular}

El estadístico-t basado en Newey y West (1987) consistente con heterocedasticidad y autocorrelación es usado para probar la hipótesis.

$*, * *$ y $* * *$ : valores significativos al $1 \%$, al $5 \%$ y al $10 \%$, respectivamente.

Fuente: elaboración propia. 
menores que cero, de las acciones en el mercado total y en los sectores al interior de este, del índice más representativo de los principales mercados de Latinoamérica, tal como se muestra en las ecuaciones 5, 6 y 7. Estas estimaciones se realizan tanto para los subperiodos de pre y poscrisis, así como para el periodo total de estudio.

\section{Modelo II precrisis}

En la tabla 4 se presenta la estimación del modelo II en los sectores de Latinoamérica para el subperiodo precrisis.

De acuerdo con los resultados presentados en la tabla 3, se observa que: en Brasil el sector bancario durante días de valorización del precio de las acciones; en Chile en el mercado total, en el sector de demás empresas durante días de rentabilidades negativas y en los sectores de alimentos y bebidas y comerciales y distribuidoras en observaciones de rentabilidades positivas; en Colombia en el mercado total, en los sectores financiero, inversiones y demás empresas en días de alza y baja de los precios y en el sector de inversiones durante días de rentabilidades mayores que cero; en Perú en el mercado total durante caídas de los precios, en el sector de servicios públicos en días de rentabilidades positivas y en el sector industrial durante caída y alza de los precios, y en Argentina en los sectores de bancos y petróleo y gas durante rentabilidades positivas, el coeficiente $\gamma 2$ se presenta negativo y estadísticamente significativo, sugiriendo una disminución en la dispersión y, por ende, presencia de efecto manada. En el mercado de México ocurre el fenómeno contrario, presentando en su mayoría coeficientes $\gamma 2$ positivos y estadísticamente significativos, indicando que los inversores divergen en sus decisiones, ocasionando un incremento más grande en la dispersión que el esperado por los modelos de valoración de activos racionales.

\section{Modelo II poscrisis}

En la tabla 5 se presenta la estimación del modelo II en los sectores de Latinoamérica para el subperiodo poscrisis.

Teniendo en cuenta los resultados presentados en la tabla 4, se puede apreciar que el coeficiente $\gamma 1$ en su mayoría es positivo y estadísticamente significativo tanto para el mercado total como para los sectores que lo componen, para todos los países objeto de estudio, confirmando la predicción de que el CSAD aumenta con el absoluto de la rentabilidad de mercado. Además, el coeficiente $\gamma 2$ es negativo y estadísticamente diferente de cero, en el mercado bursátil de Chile, en el sector de inversiones e inmobiliarias en días de disminución y aumento del precio de los activos, y en los sectores de alimentos y bebidas, comerciales y distribuidoras y servicios públicos en observaciones de rentabilidad mayores que cero; en el mercado bursátil de Colombia, en los sectores financiero y energía durante días de valorización y desvalorización del precio de los activos y en el sector de inversiones en días de rentabilidades mayores que cero; en el mercado bursátil de Perú, en el mercado total y en los sectores minero, servicios públicos y diversas durante días de rentabilidades positivas y negativas, y en el mercado bursátil de Argentina, en el sector bancario en días de aumento del precio de las acciones, sugiriendo presencia de comportamiento manada. En los mercados de Brasil y México no se presentan coeficientes $\gamma 2$ negativos y estadísticamente significativos, lo cual evidencia ausencia del efecto.

\section{Modelo II periodo total}

En la tabla 6 se presenta la estimación del modelo II en los sectores de Latinoamérica para el periodo total. 
Tabla 4

Modelo II en Latinoamérica por sectores periodo precrisis

\begin{tabular}{|c|c|c|c|c|c|c|c|c|c|}
\hline \multirow[t]{2}{*}{ Sector } & \multicolumn{3}{|c|}{ All } & \multicolumn{3}{|c|}{ Market down $(\operatorname{Ret}<0)$} & \multicolumn{3}{|c|}{ Market Up (Ret>0) } \\
\hline & A & $\gamma 1$ & $\gamma 2$ & $\alpha$ & $\gamma 1$ & $\gamma 2$ & $\alpha$ & $\gamma 1$ & $\gamma 2$ \\
\hline \multicolumn{10}{|l|}{ Brasil } \\
\hline Mercado total & $0.0145^{*}$ & $0.1166^{*}$ & $2.1761^{*}$ & $0.0144 *$ & $0.1014 *$ & $2.0597 *$ & $0.0146^{*}$ & $0.1190 * *$ & $2.7776 * * *$ \\
\hline Bancario & $0.0084^{*}$ & $0.0414 * * *$ & 0.3132 & $0.0090^{*}$ & $-0.0635 * * *$ & $2.1325^{*}$ & $0.0078 *$ & $0.1217^{*}$ & $-0.9818 * *$ \\
\hline Otros financieros & $0.0088^{*}$ & $0.1974^{*}$ & 1.2670 & $0.0089 *$ & $0.1541^{*}$ & $1.9525^{* *}$ & $0.0086^{*}$ & $0.2396^{*}$ & 0.5352 \\
\hline Construcción civil & $0.0157^{*}$ & 0.0448 & $1.8621^{*}$ & $0.0160 *$ & -0.0447 & $2.5901^{*}$ & $0.0155^{*}$ & 0.1286 & 1.1774 \\
\hline Servicios públicos & $0.0128 *$ & $0.0644 * * *$ & $1.7769 * *$ & $0.0123 *$ & 0.0791 & 1.3564 & $0.0132 *$ & 0.0533 & $2.1966 * *$ \\
\hline Consumo cíclico & $0.0067^{*}$ & $0.7178 *$ & $0.6613^{* *}$ & $0.0077 *$ & $0.5687^{*}$ & $1.4494 * *$ & $0.0069^{*}$ & $0.7522 *$ & $0.5204 * *$ \\
\hline Consumo no cíclico & $0.0119 *$ & $0.2251 *$ & -0.5172 & $0.0120 *$ & $0.1958^{*}$ & -0.3713 & $0.0119 *$ & $0.2389 *$ & -0.1408 \\
\hline Madera y papel & $0.0085^{*}$ & 0.1575 & 1.2618 & $0.0078 *$ & $0.2230 * * *$ & 0.1724 & $0.0102 *$ & -0.0356 & $5.0085^{*}$ \\
\hline Siderurgia & $0.0095^{*}$ & $0.0555 * *$ & -0.1309 & $0.0096 *$ & 0.0345 & -0.0838 & $0.0096^{*}$ & $0.0561 * *$ & 0.2196 \\
\hline Transporte & $0.0118^{*}$ & $0.1699 *$ & $3.4837 *$ & $0.0130 *$ & -0.0172 & $6.2065^{* *}$ & $0.0119 *$ & $0.1894 *$ & $3.3895^{*}$ \\
\hline Minero & $0.0056^{*}$ & $-0.0775^{* * *}$ & $3.3469 *$ & $0.0057 *$ & -0.0789 & $3.2626^{*}$ & $0.0058 *$ & -0.1153 & $4.2091 * *$ \\
\hline Telecomunicaciones & $0.0114 *$ & 0.0268 & $2.6100 * * *$ & $0.0118 *$ & -0.0790 & $4.1853 * * *$ & $0.0112 *$ & $0.1175^{* *}$ & 1.2033 \\
\hline Petroquímicos & $0.0091 *$ & 0.0423 & 1.6432 & $0.0087^{*}$ & 0.0448 & 0.7615 & $0.0094 *$ & 0.0342 & 2.9215 \\
\hline \multicolumn{10}{|l|}{ México } \\
\hline Mercado total & $0.0105^{*}$ & $0.2256^{*}$ & $3.3480 * * *$ & $0.0110 *$ & $0.1596^{*}$ & $4.2517 * *$ & $0.0101 *$ & $0.2747^{*}$ & 2.9932 \\
\hline Bienes de consumo NB & $0.0058^{*}$ & $0.3610^{*}$ & 1.8550 & $0.0064 *$ & $0.2272 * *$ & $4.6601 * * *$ & $0.0050 *$ & $0.5179^{*}$ & -1.7847 \\
\hline Materiales & $0.0093 *$ & $0.2924 * * *$ & 3.3567 & $0.0089 *$ & $0.3255^{* *}$ & 1.9590 & $0.0108 *$ & 0.0642 & $9.9834 *$ \\
\hline Alimentos, bebidas y tabaco & $0.0076^{*}$ & $0.3294 *$ & -0.6684 & $0.0074 *$ & $0.3702 *$ & -1.9065 & $0.0080 *$ & $0.2265^{*}$ & 3.5532 \\
\hline Entidades financieras & $0.0067^{*}$ & $0.1769 *$ & $6.4627 *$ & $0.0071^{*}$ & 0.1115 & $6.1493^{*}$ & $0.0081 *$ & -0.0573 & $13.4066^{*}$ \\
\hline Productos de consumo frec & $0.0076^{*}$ & 0.0144 & $6.7477 *$ & $0.0081 *$ & $-0.0725^{* * *}$ & $7.4003 *$ & $0.0058 *$ & $0.3842 *$ & -2.1775 \\
\hline Industrial & $0.0091 *$ & $0.1138 * *$ & $5.8851 *$ & $0.0097 *$ & 0.0203 & $6.3956^{*}$ & $0.0085^{*}$ & $0.2115^{*}$ & $5.3265^{*}$ \\
\hline Demás empresas & $0.0057 *$ & 0.0064 & $4.1397 * *$ & $0.0058 *$ & -0.0185 & 4.8151 & $0.0057 *$ & 0.0249 & $3.6382 * *$ \\
\hline \multicolumn{10}{|l|}{ Chile } \\
\hline Mercado total & $0.0076^{*}$ & $0.4203^{*}$ & $-1.9993 * * *$ & $0.0075^{*}$ & $0.3835^{*}$ & -1.6790 & $0.0078^{*}$ & $0.4280 *$ & -0.7075 \\
\hline Alimentos y bebidas & $0.0050 *$ & $0.5283^{*}$ & 0.0267 & $0.0053^{*}$ & $0.3769 * *$ & 4.5752 & $0.0044 *$ & $0.7745^{*}$ & $-8.8411 * *$ \\
\hline Bancarias y financieras & $0.0054 *$ & $0.3207 *$ & 3.5102 & $0.0052 *$ & $0.3074 *$ & 3.6954 & $0.0056^{*}$ & $0.3286^{*}$ & 3.5518 \\
\hline Comerciales y distribuidoras & $0.0047 *$ & $0.2716^{*}$ & -1.5940 & $0.0050 *$ & $0.1364 * *$ & 0.7306 & $0.0043 *$ & $0.4041^{*}$ & $-4.1439 * *$ \\
\hline Inversiones e inmobiliarias & $0.0045^{*}$ & $0.6138^{*}$ & -0.3124 & $0.0043^{*}$ & $0.6070^{*}$ & -2.1804 & $0.0048 *$ & $0.5615^{*}$ & $3.4360 * * *$ \\
\hline
\end{tabular}


Tabla 4 (continuación)

\begin{tabular}{|c|c|c|c|c|c|c|c|c|c|}
\hline \multirow[t]{2}{*}{ Sector } & \multicolumn{3}{|c|}{ All } & \multicolumn{3}{|c|}{ Market down $(\operatorname{Ret}<0)$} & \multicolumn{3}{|c|}{ Market Up (Ret>0) } \\
\hline & $\mathrm{A}$ & $\gamma 1$ & $\gamma 2$ & $\alpha$ & $\gamma 1$ & $\gamma 2$ & $\alpha$ & $\gamma 1$ & $\gamma 2$ \\
\hline Servicios públicos & $0.0085^{*}$ & -0.0015 & $12.1123 * *$ & $0.0088^{*}$ & -0.1045 & $13.1112 * *$ & $0.0083^{*}$ & 0.0672 & $12.2439 * *$ \\
\hline Servicios varios & $0.0057 *$ & $0.3554^{*}$ & -1.2627 & $0.0056^{*}$ & $0.2775^{*}$ & -0.3711 & $0.0060^{*}$ & $0.3810^{*}$ & 0.0686 \\
\hline Demás empresas & $0.0059^{*}$ & $0.6362 *$ & $-3.1850 * * *$ & $0.0059 *$ & $0.5632 *$ & $-3.0473 * *$ & $0.0061 *$ & $0.6469 *$ & -1.5851 \\
\hline \multicolumn{10}{|l|}{ Colombia } \\
\hline Mercado total & $0.0077^{*}$ & $0.3678^{*}$ & $-1.4370^{*}$ & $0.0076^{*}$ & $0.2934 *$ & -0.9097 & $0.0076^{*}$ & $0.4299 *$ & $-1.6948^{*}$ \\
\hline Financiero & $0.0046^{*}$ & $0.4598^{*}$ & $-1.6405^{*}$ & $0.0047 *$ & $0.4164 *$ & $-1.6976^{* * * *}$ & $0.0046^{*}$ & $0.4891 *$ & $-1.3940 * *$ \\
\hline Inversiones & $0.0048 *$ & $0.3743^{*}$ & $-1.4677 *$ & $0.0049 *$ & $0.3043^{*}$ & $-0.8790 * * *$ & $0.0048 *$ & $0.4222 *$ & $-1.7035^{*}$ \\
\hline Energía & $0.0036^{*}$ & $0.3705^{*}$ & $-1.4177 *$ & $0.0039 *$ & $0.2992 *$ & -0.5775 & $0.0035^{*}$ & $0.4113 *$ & $-1.8660^{*}$ \\
\hline Demás empresas & $0.0063 *$ & $0.5973^{*}$ & $-3.5006^{*}$ & $0.0068 *$ & $0.4550^{*}$ & $-2.5352 *$ & $0.0059 *$ & $0.7155^{*}$ & $-4.1937 *$ \\
\hline \multicolumn{10}{|l|}{ Perú } \\
\hline Mercado total & $0.0089 *$ & $0.8211 *$ & $-4.5108^{*}$ & $0.0094 *$ & $0.7450 *$ & $-4.2022 * *$ & $0.0086^{*}$ & $0.8335^{*}$ & -2.8032 \\
\hline Mineras & $0.0092 *$ & $0.3489 *$ & $4.2012 *$ & $0.0102 *$ & $0.2146^{* * *}$ & $5.2549 *$ & $0.0083^{*}$ & $0.4610 *$ & $3.3022 *$ \\
\hline Servicios públicos & $0.0035^{*}$ & $0.6069 *$ & $-2.5092 * * *$ & $0.0040 *$ & $0.5789 *$ & -1.9463 & $0.0028 *$ & $0.6760 *$ & $-4.4992 *$ \\
\hline Industrial & $0.0075^{*}$ & $0.8076^{*}$ & $-4.2265^{*}$ & $0.0086^{*}$ & $0.6223^{*}$ & $-2.6351 * * *$ & $0.0067 *$ & $0.9207 *$ & $-4.5109 *$ \\
\hline Diversas & $0.0042 *$ & $0.8064 *$ & -1.4842 & $0.0046^{*}$ & $0.7867 *$ & -1.1924 & $0.0037 * *$ & $0.8386^{*}$ & -1.8826 \\
\hline \multicolumn{10}{|l|}{ Argentina } \\
\hline Mercado total & $0.0128 *$ & $0.2177 *$ & $4.0979 *$ & $0.0125^{*}$ & $0.2517 *$ & 1.9577 & $0.0121 *$ & $0.3146^{*}$ & $3.7936^{*}$ \\
\hline Metalúrgica y siderúrgica & $0.0083^{*}$ & $0.2069 *$ & 0.3730 & $0.0085^{*}$ & 0.1054 & 1.2538 & $0.0081 *$ & $0.2889 *$ & -0.1664 \\
\hline Petróleo y gas & $0.0072 *$ & $0.5139 *$ & $-1.7708 *$ & $0.0084 *$ & $0.3160 *$ & $3.1818 * *$ & $0.0076 *$ & $0.4696^{*}$ & $-1.7780 *$ \\
\hline Bancos & $0.0067^{*}$ & $0.2272 *$ & -0.2405 & $0.0082 *$ & 0.0410 & $1.5907 * *$ & $0.0064 *$ & $0.2841 *$ & $-0.4597 * * *$ \\
\hline Demás empresas & $0.0099 *$ & $0.2312 *$ & $2.6575^{*}$ & $0.0095 *$ & $0.1551 * *$ & $3.5469 *$ & $0.0107 *$ & $0.2631 * *$ & $2.2593 * *$ \\
\hline
\end{tabular}

El estadístico-t basado en Newey y West (1987) consistente con heterocedasticidad y autocorrelación es usado para probar la hipótesis.

$*$, ** y ***: valores significativos al $1 \%$, al $5 \%$ y al $10 \%$, respectivamente.

Fuente: elaboración propia. 
Tabla 5

Modelo II en Latinoamérica por sectores periodo poscrisis

\begin{tabular}{|c|c|c|c|c|c|c|c|c|c|}
\hline \multirow[t]{2}{*}{ Sector } & \multicolumn{3}{|c|}{ All } & \multicolumn{3}{|c|}{ Market down $(\operatorname{Ret}<0)$} & \multicolumn{3}{|c|}{ Market Up $(\operatorname{Ret}>0)$} \\
\hline & $\alpha$ & $\gamma 1$ & $\gamma 2$ & $\alpha$ & $\gamma 1$ & $\gamma 2$ & $\alpha$ & $\gamma 1$ & $\gamma 2$ \\
\hline \multicolumn{10}{|l|}{ Brasil } \\
\hline Mercado total & $0.0121 *$ & $0.2417^{*}$ & $1.0660 * * *$ & $0.0125^{*}$ & $0.1859^{*}$ & $1.7769 *$ & $0.0119 *$ & $0.2844^{*}$ & 0.6259 \\
\hline Bancario & $0.0055^{*}$ & $0.1118^{*}$ & -0.0303 & $0.0059 *$ & $0.0540 * *$ & $0.6222 *$ & $0.0054 *$ & $0.1431 *$ & -0.2622 \\
\hline Otros financieros & $0.0095^{*}$ & $0.2761^{*}$ & -0.1064 & $0.0095^{*}$ & $0.2805^{*}$ & 0.3113 & $0.0097 *$ & $0.2523^{*}$ & -0.1039 \\
\hline Construcción civil & $0.0128 *$ & 0.0220 & $1.9787^{*}$ & $0.0123 *$ & 0.0288 & $1.6661 *$ & $0.0136^{*}$ & -0.0142 & $2.7694 *$ \\
\hline Servicios públicos & $0.0089 *$ & $0.2668^{*}$ & -0.1060 & $0.0095 *$ & $0.1890^{* * *}$ & 2.4369 & $0.0088^{*}$ & $0.2563 *$ & -0.4372 \\
\hline Consumo cíclico & $0.0102 *$ & $0.2279 *$ & 1.4605 & $0.0110 *$ & 0.1137 & $3.7511 *$ & $0.0100 *$ & $0.2599 *$ & 0.9314 \\
\hline Consumo no cíclico & $0.0116^{*}$ & $0.2045^{*}$ & $2.4107 *$ & $0.0119 *$ & $0.1557 *$ & $2.7451 *$ & $0.0113^{*}$ & $0.2423^{*}$ & $2.2145 * *$ \\
\hline Madera y papel & $0.0108 *$ & -0.0617 & 3.5544 & $0.0107 *$ & -0.0401 & 1.9133 & $0.0104^{*}$ & -0.0188 & 3.8543 \\
\hline Siderurgia & $0.0071 *$ & $0.1102 *$ & -0.1295 & $0.0072 *$ & $0.0771 * *$ & -0.0787 & $0.0070^{*}$ & $0.1438^{*}$ & -0.2576 \\
\hline Transporte & $0.0110^{*}$ & $0.2679 *$ & 0.1769 & $0.0111 *$ & $0.2011 *$ & 0.5868 & $0.0108 *$ & $0.3289^{*}$ & -0.1785 \\
\hline Minero & $0.0070^{*}$ & $0.2560^{*}$ & $1.9125^{*}$ & $0.0075^{*}$ & $0.2511^{*}$ & 0.7159 & $0.0057 *$ & $0.3551 *$ & $1.7476^{*}$ \\
\hline Telecomunicaciones & $0.0082 *$ & $0.2371 *$ & $2.6055^{* *}$ & $0.0081^{*}$ & $0.2672 *$ & $2.5620 * * *$ & $0.0082 *$ & $0.2043^{* *}$ & $2.7015 * * *$ \\
\hline Petroquímicos & $0.0070^{*}$ & $0.1103 *$ & 0.8633 & $0.0078 *$ & 0.0140 & $1.5822 * *$ & $0.0064 *$ & $0.1989^{*}$ & 0.2772 \\
\hline \multicolumn{10}{|l|}{ México } \\
\hline Mercado total & $0.0100 *$ & $0.2734 *$ & $2.7355^{*}$ & $0.0102 *$ & $0.2478^{*}$ & 2.0515 & $0.0097 *$ & $0.3295^{*}$ & $2.5589 *$ \\
\hline Bienes de consumo NB & $0.0063^{*}$ & $0.4272 *$ & -0.3044 & $0.0059 *$ & $0.4884^{*}$ & -1.2700 & $0.0066^{*}$ & $0.3965^{*}$ & 0.0048 \\
\hline Materiales & $0.0090^{*}$ & $0.2996^{*}$ & -0.2706 & $0.0092 *$ & $0.2747^{*}$ & -0.0136 & $0.0088^{*}$ & $0.3185^{*}$ & -0.4157 \\
\hline Alimentos, bebidas y tabaco & $0.0087^{*}$ & 0.0234 & $11.4578^{*}$ & $0.0094 *$ & $-0.1755^{* *}$ & $12.7489 *$ & $0.0089^{*}$ & 0.0055 & $16.4472 *$ \\
\hline Entidades financieras & $0.0105^{*}$ & -0.0303 & $8.1937 * *$ & $0.0085^{*}$ & $0.2944^{*}$ & -0.1040 & $0.0111 *$ & -0.0906 & $10.2633^{*}$ \\
\hline Productos de consumo frec & $0.0080 *$ & $0.0984 * * *$ & $6.8121 *$ & $0.0073 *$ & $0.1926^{* * *}$ & 2.8208 & $0.0080 *$ & $0.1488 * *$ & $6.7794 *$ \\
\hline Industrial & $0.0090^{*}$ & $0.2139 *$ & $2.2690 *$ & $0.0091 *$ & $0.2248^{*}$ & 1.1901 & $0.0088^{*}$ & $0.2468^{*}$ & $2.2533^{*}$ \\
\hline Demás empresas & $0.0069 *$ & $0.2479 *$ & 2.2015 & $0.0064 *$ & $0.3259^{*}$ & -0.7735 & $0.0071^{*}$ & $0.2452 *$ & 2.8838 \\
\hline \multicolumn{10}{|l|}{ Chile } \\
\hline Mercado total & $0.0078 *$ & $0.3204^{*}$ & 0.5943 & $0.0081 *$ & $0.2165^{*}$ & 0.3317 & $0.0074^{*}$ & $0.4522 *$ & -0.2812 \\
\hline Alimentos y bebidas & $0.0058 *$ & $0.3840^{*}$ & -0.3482 & $0.0062 *$ & $0.2410^{*}$ & $4.4804 * * *$ & $0.0057 *$ & $0.4410^{*}$ & $-1.5606^{* *}$ \\
\hline Bancarias y financieras & $0.0065^{*}$ & $0.2104 *$ & 0.2028 & $0.0065^{*}$ & $0.1698^{*}$ & 0.1853 & $0.0064^{*}$ & $0.2507^{*}$ & -0.0221 \\
\hline Comerciales y distribuidoras & $0.0062 *$ & $0.1375^{*}$ & -0.6480 & $0.0062 *$ & 0.0541 & -0.0209 & $0.0062 *$ & $0.2123^{*}$ & $-1.3339 * *$ \\
\hline Inversiones e inmobiliarias & $0.0065^{*}$ & $0.4572 *$ & $-3.5342 *$ & $0.0063 *$ & $0.3802 *$ & $-2.8092 *$ & $0.0067 *$ & $0.5206^{*}$ & $-4.1552 * *$ \\
\hline Servicios públicos & $0.0064 *$ & $0.2613^{*}$ & $-0.9223^{*}$ & $0.0065^{*}$ & $0.1635^{*}$ & 1.4163 & $0.0065^{*}$ & $0.3062 *$ & $-1.3325^{*}$ \\
\hline Servicios varios & $0.0070^{*}$ & $0.2157^{*}$ & $3.4341^{*}$ & $0.0063^{*}$ & $0.2821^{*}$ & -0.2111 & $0.0072 *$ & $0.2703^{*}$ & $3.3042 *$ \\
\hline
\end{tabular}


Tabla 5 (continuación)

\begin{tabular}{|c|c|c|c|c|c|c|c|c|c|}
\hline \multirow[t]{2}{*}{ Sector } & \multicolumn{3}{|c|}{ All } & \multicolumn{3}{|c|}{ Market down $(\operatorname{Ret}<0)$} & \multicolumn{3}{|c|}{ Market Up (Ret>0) } \\
\hline & $\alpha$ & $\gamma 1$ & $\gamma 2$ & $\alpha$ & $\gamma 1$ & $\gamma 2$ & $\alpha$ & $\gamma 1$ & $\gamma 2$ \\
\hline Demás empresas & $0.0080^{*}$ & $0.3343 *$ & 1.4266 & $0.0083 *$ & $0.2666^{*}$ & 0.4348 & $0.0078 *$ & $0.3856^{*}$ & 2.7229 \\
\hline \multicolumn{10}{|l|}{ Colombia } \\
\hline Mercado total & $0.0081^{*}$ & $0.3069 *$ & 1.2950 & $0.0086^{*}$ & 0.1727 & 5.4099 & $0.0078^{*}$ & $0.3750 *$ & -0.9329 \\
\hline Financiero & $0.0057^{*}$ & $0.3376^{*}$ & $-2.4697 *$ & $0.0059 *$ & $0.2937^{*}$ & $-2.1260 *$ & $0.0055^{*}$ & $0.3781 *$ & $-2.7839^{*}$ \\
\hline Inversiones & $0.0052 *$ & $0.2942 *$ & $-1.5962 * * *$ & $0.0053^{*}$ & $0.2625^{*}$ & -0.9149 & $0.0051^{*}$ & $0.3163^{*}$ & $-1.9885^{* * *}$ \\
\hline Petróleo & $0.0075^{*}$ & $0.2165 *$ & $3.4022 *$ & $0.0080^{*}$ & $0.1841^{*}$ & $3.5474^{*}$ & $0.0071^{*}$ & $0.2435 * *$ & 3.4959 \\
\hline Energía & $0.0048^{*}$ & $0.3782 *$ & $-4.2450 *$ & $0.0047 *$ & $0.3360 *$ & $-2.6732 * * *$ & $0.0050^{*}$ & $0.3875^{*}$ & $-4.6152 *$ \\
\hline Demás empresas & $0.0092^{*}$ & $0.2080 *$ & $9.6708 *$ & $0.0092 *$ & 0.1170 & $10.2832 *$ & $0.0091^{*}$ & $0.2815^{*}$ & $9.4906^{*}$ \\
\hline \multicolumn{10}{|l|}{ Perú } \\
\hline Mercado total & $0.0099 *$ & $0.4819^{*}$ & $-2.1747 *$ & $0.0134 *$ & 0.0114 & 2.0310 & $0.0103^{*}$ & $0.4255^{*}$ & -0.6718 \\
\hline Mineras & $0.0116^{*}$ & $0.4342 *$ & $-2.1156^{*}$ & $0.0111 *$ & $0.4233 *$ & $-2.0148^{*}$ & $0.0119^{*}$ & $0.4516^{*}$ & $-2.3028 *$ \\
\hline Servicios públicos & $0.0041^{*}$ & $0.4526^{*}$ & $-2.3110 *$ & $0.0046^{*}$ & $0.4242 *$ & $-2.5004^{*}$ & $0.0037 *$ & $0.4640^{*}$ & $-1.7358 *$ \\
\hline Industrial & $0.0093^{*}$ & $0.4214 *$ & -1.5186 & $0.0090 *$ & $0.4228 *$ & -1.5232 & $0.0096^{*}$ & $0.4197 *$ & -1.4968 \\
\hline Diversas & $0.0050 *$ & $0.4877 *$ & $-3.1870 *$ & $0.0051^{*}$ & $0.4628 *$ & $-2.9455^{*}$ & $0.0047 *$ & $0.5407 *$ & $-4.1097 *$ \\
\hline \multicolumn{10}{|l|}{ Argentina } \\
\hline Mercado total & $0.0127 *$ & $0.1656^{*}$ & 1.4587 & $0.0128 *$ & $0.0913 * * *$ & $1.9107 * * *$ & $0.0128 *$ & $0.1835^{*}$ & $2.2202 *$ \\
\hline Metalúrgica y siderúrgica & $0.0084 *$ & $0.1940^{*}$ & -0.1686 & $0.0086^{*}$ & $0.1163 *$ & 0.3579 & $0.0083^{*}$ & $0.2525^{*}$ & -0.3948 \\
\hline Petróleo y gas & $0.0089 *$ & $0.1979 *$ & $2.6950 * *$ & $0.0083^{*}$ & $0.1930 * * *$ & 2.5270 & $0.0096^{*}$ & $0.1896^{*}$ & $3.1997 *$ \\
\hline Bancos & $0.0088^{*}$ & $0.0456^{* *}$ & 0.3656 & $0.0094 *$ & -0.0262 & $0.9679 *$ & $0.0079 *$ & $0.1303 *$ & $-0.5061 * * *$ \\
\hline Demás empresas & $0.0111^{*}$ & $0.2190^{*}$ & $1.0139 * *$ & $0.0113^{*}$ & 0.0715 & $1.9567^{*}$ & $0.0107 *$ & $0.3769^{*}$ & -0.5384 \\
\hline
\end{tabular}

El estadístico-t basado en Newey y West (1987) consistente con heterocedasticidad y autocorrelación es usado para probar la hipótesis.

$*, * *$ y $* * *$ : valores significativos al $1 \%$, al $5 \%$ y al $10 \%$, respectivamente.

Fuente: elaboración propia. 
Tabla 6

Modelo II en Latinoamérica por sectores periodo total

\begin{tabular}{|c|c|c|c|c|c|c|c|c|c|}
\hline \multirow[t]{2}{*}{ Cartera } & \multicolumn{3}{|c|}{ All } & \multicolumn{3}{|c|}{ Market down $($ Ret<0) } & \multicolumn{3}{|c|}{ Market Up (Ret>0) } \\
\hline & $\alpha$ & $\gamma 1$ & $\gamma 2$ & $\alpha$ & $\gamma 1$ & $\gamma 2$ & $\alpha$ & $\gamma 1$ & $\gamma 2$ \\
\hline \multicolumn{10}{|l|}{ Brasil } \\
\hline Mercado total & $0.0133^{*}$ & $0.1840 *$ & $1.5140 *$ & $0.0134^{*}$ & $0.1424 *$ & $1.9728 *$ & $0.0132 *$ & $0.2205 *$ & $1.2036 * * *$ \\
\hline Bancario & $0.0070^{*}$ & $0.0801 *$ & 0.1022 & $0.0074^{*}$ & 0.0121 & $1.0036^{*}$ & $0.0068^{*}$ & $0.1153 *$ & $-0.2185^{* *}$ \\
\hline Otros financieros & $0.0092 *$ & $0.2351 *$ & 0.3577 & $0.0094^{*}$ & $0.1899 *$ & $1.2769 * *$ & $0.0091^{*}$ & $0.2489 *$ & 0.0135 \\
\hline Construcción civil & $0.0138^{*}$ & 0.0275 & $2.0083 *$ & $0.0135^{*}$ & 0.0113 & $1.9521 *$ & $0.0143^{*}$ & 0.0364 & $2.1625^{*}$ \\
\hline Servicios públicos & $0.0107 *$ & $0.1806^{*}$ & 0.5149 & $0.0107 *$ & $0.1522 *$ & 1.1152 & $0.0107 *$ & $0.1937 *$ & 0.2527 \\
\hline Consumo cíclico & $0.0073^{*}$ & $0.5785^{*}$ & $0.9856^{*}$ & $0.0085^{*}$ & $0.4453 *$ & $1.8727 *$ & $0.0073^{*}$ & $0.6011 *$ & $0.8354 *$ \\
\hline Consumo no cíclico & $0.0120^{*}$ & $0.1728^{*}$ & $1.8588 *$ & $0.0122 *$ & $0.1309^{*}$ & $2.0845^{* *}$ & $0.0118^{*}$ & $0.2056^{*}$ & $1.8881 *$ \\
\hline Madera y papel & $0.0104^{*}$ & -0.0273 & 3.2073 & $0.0103 *$ & -0.0125 & $2.1799 * * *$ & $0.0104 *$ & -0.0186 & 3.9827 \\
\hline Siderurgia & $0.0084^{*}$ & $0.0763^{*}$ & -0.0363 & $0.0085^{*}$ & $0.0513 * *$ & -0.0314 & $0.0084^{*}$ & $0.0974 *$ & -0.0026 \\
\hline Transporte & $0.0123^{*}$ & $0.1102 *$ & $3.4917 *$ & $0.0118 *$ & 0.1257 & 2.9057 & $0.0124 *$ & $0.1361 *$ & $3.4488 *$ \\
\hline Minero & $0.0054^{*}$ & $0.1494 *$ & $2.1195^{*}$ & $0.0063^{*}$ & 0.0956 & $2.1452 *$ & $0.0045^{*}$ & $0.2082 *$ & $2.0227 *$ \\
\hline Telecomunicaciones & $0.0101 *$ & $0.0869 * *$ & $3.0935^{*}$ & $0.0102 *$ & 0.0546 & $3.5119 *$ & $0.0099^{*}$ & $0.1228 * *$ & $2.6003 * *$ \\
\hline Petroquímicos & $0.0081^{*}$ & $0.0825^{*}$ & $1.1066^{* * *}$ & $0.0083^{*}$ & 0.0202 & $1.3692 * *$ & $0.0078^{*}$ & $0.1441 *$ & 0.9552 \\
\hline \multicolumn{10}{|l|}{ México } \\
\hline Mercado total & $0.0105^{*}$ & $0.1943 *$ & $4.7510^{*}$ & $0.0112^{*}$ & 0.0673 & $6.9241^{*}$ & $0.0099 *$ & $0.3021 *$ & $2.8250^{*}$ \\
\hline Bienes de consumo NB & $0.0059^{*}$ & $0.4246^{*}$ & -0.0517 & $0.0061^{*}$ & $0.3765^{*}$ & 1.1006 & $0.0059^{*}$ & $0.4357 *$ & -0.3341 \\
\hline Materiales & $0.0090^{*}$ & $0.3296^{*}$ & 0.7185 & $0.0092 *$ & $0.2779 * *$ & 1.4552 & $0.0089 *$ & $0.3672 *$ & 0.2022 \\
\hline Alimentos, bebidas y tabaco & $0.0087^{*}$ & 0.0290 & $11.0227 *$ & $0.0092 *$ & -0.0947 & $11.8038^{*}$ & $0.0087^{*}$ & 0.0074 & $15.2720^{*}$ \\
\hline Entidades financieras & $0.0084^{*}$ & $0.0932 * * *$ & $7.1272 *$ & $0.0084^{*}$ & $0.0796 * * *$ & $6.0663^{*}$ & $0.0093^{*}$ & -0.0243 & $10.7476 *$ \\
\hline Productos de consumo frec & $0.0059^{*}$ & $0.3447 *$ & $2.0689 *$ & $0.0061^{*}$ & $0.2887 * *$ & $2.1480 *$ & $0.0075^{*}$ & $0.1197 * *$ & $6.7934 *$ \\
\hline Industrial & $0.0091 *$ & $0.1537 *$ & $4.6356^{*}$ & $0.0097 *$ & 0.0492 & $5.6673^{*}$ & $0.0085^{*}$ & $0.2407 *$ & $3.9757 *$ \\
\hline Demás empresas & $0.0066^{*}$ & 0.0630 & $3.8806^{*}$ & $0.0063 *$ & 0.0894 & 2.9643 & $0.0068^{*}$ & 0.0524 & $4.3133 *$ \\
\hline \multicolumn{10}{|l|}{ Chile } \\
\hline Mercado total & $0.0078^{*}$ & $0.3547^{*}$ & -0.0407 & $0.0078^{*}$ & $0.2952 *$ & -0.2488 & $0.0076^{*}$ & $0.4319 *$ & -0.1401 \\
\hline Alimentos y bebidas & $0.0054^{*}$ & $0.4682 *$ & -0.0064 & $0.0058^{*}$ & $0.2956^{* *}$ & 5.5207 & $0.0051^{*}$ & $0.5973 *$ & $-4.2626^{*}$ \\
\hline Bancarias y financieras & $0.0056^{*}$ & $0.3325^{*}$ & -1.0140 & $0.0055^{*}$ & $0.3337 *$ & -1.6472 & $0.0058^{*}$ & $0.3345^{*}$ & -0.4945 \\
\hline Comerciales y distribuidoras & $0.0054^{*}$ & $0.2088^{*}$ & $-1.0131 * *$ & $0.0057 *$ & $0.0838 * *$ & 1.0348 & $0.0052 *$ & $0.3059 *$ & $-2.4380^{*}$ \\
\hline Inversiones e inmobiliarias & $0.0054^{*}$ & $0.5394 *$ & -1.4391 & $0.0052^{*}$ & $0.4964 *$ & -1.9161 & $0.0056^{*}$ & $0.5465 *$ & -0.0708 \\
\hline Servicios públicos & $0.0069 *$ & $0.2735^{*}$ & 2.4879 & $0.0079 *$ & -0.0557 & $11.4462 * *$ & $0.0066^{*}$ & $0.4031 *$ & -0.8385 \\
\hline
\end{tabular}


Tabla 6 (continuación)

\begin{tabular}{|c|c|c|c|c|c|c|c|c|c|}
\hline \multirow[t]{2}{*}{ Cartera } & \multicolumn{3}{|c|}{ All } & \multicolumn{3}{|c|}{ Market down $(\operatorname{Ret}<0)$} & \multicolumn{3}{|c|}{ Market Up (Ret>0) } \\
\hline & $\alpha$ & $\gamma 1$ & $\gamma 2$ & $\alpha$ & $\gamma 1$ & $\gamma 2$ & $\alpha$ & $\gamma 1$ & $\gamma 2$ \\
\hline Servicios varios & $0.0069^{*}$ & $0.1913^{*}$ & $3.4892 *$ & $0.0061^{*}$ & $0.2708^{*}$ & -0.1371 & $0.0070^{*}$ & $0.2718^{*}$ & $3.3016^{*}$ \\
\hline Demás empresas & $0.0069 *$ & $0.4819^{*}$ & -0.6973 & $0.0070^{*}$ & $0.4148^{*}$ & -1.2173 & $0.0069^{*}$ & $0.5127 *$ & 0.8074 \\
\hline \multicolumn{10}{|l|}{ Colombia } \\
\hline Mercado total & $0.0078 *$ & $0.3711^{*}$ & $-1.4736^{*}$ & $0.0078^{*}$ & $0.3217 *$ & $-1.2210 * *$ & $0.0076^{*}$ & $0.4183^{*}$ & $-1.5964 *$ \\
\hline Financiero & $0.0051 *$ & $0.4110^{*}$ & $-1.5289 *$ & $0.0053^{*}$ & $0.3607 *$ & -1.4544 & $0.0050 *$ & $0.4469^{*}$ & $-1.3951 *$ \\
\hline Inversiones & $0.0050^{*}$ & $0.3380 *$ & $-1.2780 *$ & $0.0051^{*}$ & $0.2883^{*}$ & $-0.8802 * * *$ & $0.0049^{*}$ & $0.3819 *$ & $-1.4864 *$ \\
\hline Petróleo & $0.0075^{*}$ & $0.2165^{*}$ & $3.4022 *$ & $0.0080^{*}$ & $0.1841^{*}$ & $3.5474 *$ & $0.0071 *$ & $0.2435^{* *}$ & 3.4959 \\
\hline Energía & $0.0044 *$ & $0.3304 *$ & $-1.2530 *$ & $0.0044 *$ & $0.2819 *$ & -0.5332 & $0.0044 *$ & $0.3568 *$ & $-1.6600 *$ \\
\hline Demás empresas & $0.0082^{*}$ & $0.3515^{* *}$ & 2.3418 & $0.0091^{*}$ & 0.1237 & 5.3104 & $0.0073 *$ & $0.5819 *$ & -1.1016 \\
\hline \multicolumn{10}{|l|}{ Perú } \\
\hline Mercado total & $0.0095^{*}$ & $0.6489 *$ & $-3.3195^{*}$ & $0.0097^{*}$ & $0.5960 *$ & $-2.9597 *$ & $0.0093 *$ & $0.6916 *$ & $-3.3544 * * *$ \\
\hline Minero & $0.0107 *$ & $0.3339 *$ & $2.6859 * *$ & $0.0106^{*}$ & $0.3062 *$ & 2.5217 & $0.0108 *$ & $0.3327 *$ & $3.3589 *$ \\
\hline Servicios públicos & $0.0037^{*}$ & $0.5565^{*}$ & $-3.0494 *$ & $0.0042 *$ & $0.5431 *$ & $-3.1913^{*}$ & $0.0033 *$ & $0.5626^{*}$ & $-2.6753 *$ \\
\hline Industrial & $0.0084 *$ & $0.6225^{*}$ & $-3.0091^{*}$ & $0.0088^{*}$ & $0.5220^{*}$ & $-2.1752 *$ & $0.0079 *$ & $0.7279 *$ & $-4.0446 * *$ \\
\hline Diversas & $0.0039^{*}$ & $0.7343^{*}$ & -1.9721 & $0.0037^{*}$ & $0.7617^{*}$ & -2.5923 & $0.0041^{*}$ & $0.6842 *$ & -0.8780 \\
\hline \multicolumn{10}{|l|}{ Argentina } \\
\hline Mercado total & $0.0130 *$ & $0.1632 *$ & $3.4268^{*}$ & $0.0124^{*}$ & $0.2129^{*}$ & 1.2702 & $0.0128 *$ & $0.1916^{*}$ & $4.3625^{*}$ \\
\hline Metalúrgica y siderúrgica & $0.0083^{*}$ & $0.2109 *$ & -0.2003 & $0.0109 *$ & 0.0282 & 0.0618 & $0.0082 *$ & $0.2774 *$ & -0.5033 \\
\hline Petróleo y gas & $0.0078 *$ & $0.3857 *$ & -0.0081 & $0.0081 *$ & $0.2891 *$ & 1.9125 & $0.0080 *$ & $0.4062 *$ & -0.7007 \\
\hline Bancario & $0.0075^{*}$ & $0.1524 *$ & -0.0210 & $0.0086^{*}$ & 0.0182 & $1.3045^{* *}$ & $0.0072 *$ & $0.1939 *$ & -0.2169 \\
\hline Demás empresas & $0.0108 *$ & $0.1808 *$ & $2.7595^{*}$ & $0.0107 *$ & 0.0787 & $3.5678 *$ & $0.0113 *$ & $0.2441 *$ & $2.3094 * *$ \\
\hline
\end{tabular}

El estadístico-t basado en Newey y West (1987) consistente con heterocedasticidad y autocorrelación es usado para probar la hipótesis.

$*$, ** y ***: valores significativos al $1 \%$, al $5 \%$ y al $10 \%$, respectivamente.

Fuente: elaboración propia. 
De acuerdo con los resultados presentados en la tabla 5, se aprecia que el coeficiente $\gamma 2$ es negativo y estadísticamente significativo: en el IBOVESPA en el sector bancario durante días de rentabilidades mayores que cero; en el IPSA en los sectores de alimentos y bebidas y comerciales y distribuidoras durante días de aumento del precio de las acciones; en el COLCAP en el mercado total y en el sector de inversiones durante días de aumento y disminución en los precios, y en los sectores financiero y energía en días de rentabilidades positivas; y en el IGBVL en el mercado total y en los sectores industrial y servicios públicos en días de rentabilidades positivas y negativas. Los resultados anteriores sugieren una reducción en el nivel de dispersión y, por lo tanto, una convergencia en las decisiones de los inversores, que conduce a comportamiento manada. Respecto a los mercados de Argentina y México, no existe evidencia de efecto manada; sin embargo, se puede resaltar que en este último los coeficientes en su mayoría son positivos y estadísticamente significativos, lo que sugiere un aumento de la dispersión mayor a la esperada (comportamiento anti-manada) y, por lo tanto, una divergencia marcada en las decisiones de inversión de los participantes del mercado.

\section{Conclusiones}

A partir de los resultados obtenidos mediante el modelo I propuesto por Christie y Huang (1995), no se halló evidencia alguna de efecto manada en el periodo total (02/01/2002-30/06/2014), así como tampoco en los periodos de pre y poscrisis subprime en los principales mercados bursátiles de América Latina (Argentina, Brasil, Chile, Colombia, México y Perú). Iguales resultados se obtienen al analizar este efecto en los sectores que componen los diferentes índices bajo estudio.

Sin embargo, al utilizar un segundo modelo más sensible para detectar el efecto manada, planteado por Chang et al. (2000), se detectó este comportamiento en algunos países de Latinoamérica, así como también en sectores al interior de ellos, tanto en los subperiodos pre y poscrisis como en el periodo total.

El ranking de mayor a menor presencia de efecto manada en Latinoamérica estaría dado por Colombia (19) ${ }^{1}$, Perú (16), Chile (10), Argentina (3), Brasil (2) y México (0). Al matizar el ranking por subperiodos, en el periodo precrisis la clasificación de mayor a menor efecto estaría dada por Colombia (8), Perú (4), Chile (3), Argentina (2), Brasil (1) y México (0), mientras que en el periodo poscrisis la clasificación sería: Perú (6), Colombia (5) y Chile (5), Argentina (1), Brasil (0) y México (0). De estas clasificaciones se puede concluir que los países donde más se evidencia la presencia de efecto manada son Colombia, Chile y Perú, lo que podría interpretarse como que los inversores se anticiparon a la crisis del 2008 moviendo sus capitales hacia estos mercados latinoamericanos y, por tal motivo, generando el efecto estudiado; además, se debe destacar que en el periodo de precrisis Colombia lideraba el ranking del efecto, mientras que, después de este periodo, Perú pasó a ocupar esta posición, lo que dejaría entrever que en un comienzo el país de preferencia fue Colombia, pero que después de 2008 se privilegió a Perú como la economía más atractiva.

Se evidencia que el efecto manada se presenta con mayor regularidad cuando los mercados latinoamericanos están al alza, lo que sugiere que los inversores tienden a presentar este comportamiento como respuesta a las buenas noticias, contrario a lo planteado por Chen (2013), quien sostiene que este efecto se presenta cuando el mercado está a la baja, en respuesta a las malas noticias.

\footnotetext{
${ }^{1}$ El número entre paréntesis representa las veces que el efecto manada se presenta en el respectivo mercado.
} 
Si se evaluara la eficiencia del mercado a partir de los resultados del efecto manada presentados en este trabajo, los mercados bursátiles de México y Brasil serían los que mejor cumplirían este supuesto, mientras que Colombia, Perú y Chile cumplen en menor grado esta hipótesis. Los resultados para Argentina son comprensibles teniendo en cuenta que, al ser una economía fuertemente intervenida por el Estado, no genera confianza en los inversores como para producir un efecto manada en su mercado bursátil.

De los resultados de la presente investigación se podría inferir que el modelo II planteado por Chang et al. (2000) es mejor que el modelo I para detectar la presencia de efecto manada. Sin embargo, para llegar a esta conclusión de forma definitiva, deberían contrastarse las 2 metodologías en un mayor número de mercados (mundial).

Dado que el número de empresas al interior de algunos de los sectores e índices bursátiles latinoamericanos es relativamente pequeño, y que adicionalmente los modelos de regresión lineal utilizados parten de supuestos que se asumen como ciertos, los resultados de la presente investigación deben ser tomados con cierto grado de precaución, evitando caer en generalizaciones. Además, es importante destacar que el objetivo del presente estudio es verificar empíricamente la presencia o no de efecto manada en la región, pero no contempla determinar las causas (racionales o irracionales) que originan este comportamiento en los inversores.

\section{Referencias}

Autorregulador del Mercado de Valores de Colombia, AMV. (2009). Impacto de la crisis financiera internacional en el sistema financiero colombiano y en su regulación [Documento de Investigación]. Bogotá.

Bikhchandani, S. y Sharma, S. (2001). Herd behavior in financial markets. IMF Staff Papers, International Monetary Fund, 47, 279-310.

Borensztein, E. R. y Gelos, R. G. (2003). Leaders and followers: Emerging market fund behavior during tranquil and turbulent times. Emerging Markets Review, 25-38.

Caporale, G. M., Economou, F. y Philippas, N. (2008). Herding behaviour in extreme market conditions: The case of the Athens Stock Exchange. Economics Bulletin, 7(17), 1-13.

Chang, E. C., Cheng, J. W. y Khorana, A. (2000). An examination of herd behavior in equity markets: An international perspective. Journal of Banking \& Finance, 24, 1651-1679.

Chen, T. (2013). Do investors herd in global stock markets? Journal of Behavioral Finance, 14, 230-239.

Chiang, T. C. y Zheng, D. (2010). An empirical analysis of herd behavior in global stock markets. Journal of Banking \& Finance, 34, 1911-1921.

Christie, W. G. y Huang, R. D. (1995). Following the pied piper: Do individual returns herd around the market. Financial Analysts Journal, 31-37.

De Almeida, R. P., Costa, H. C. y da Costa, N. C., Jr. (2012). Herd behavior in Latin American stock markets. Latin American Business Rewiew, 13(2), 81-102.

Demirer, R. y Kutan, A. M. (2006). Does herding behavior exist in Chinese stock markets? International Financial Markets, Institutions \& Money, 16(2), 123-142.

Devenow, A. y Welch, I. (1996). Rational herding in financial economics. European Economic Review, 40, 603-615.

Economou, F., Kostakis, A. y Philippas, N. (2011). Cross-country effects in herding behaviour: Evidence from four south European markets. Journal of International Financial Markets, Institutions \& Money, 21(3), 443-460.

Fama, E. F. (1970). Efficient capital markets: A review of theory and empirical work. The Journal of Finance, 25(2), $383-417$.

Gebka, B. y Wohar, M. E. (2013). International herding: Does it differ across sectors? Journal of International Financial Markets, Institutions \& Money, 23, 55-84.

Gujarati, D. N. y Porter, D. C. (2010). Econometría. México D.F: McGraw Hill.

Henker, J., Henker, T. y Mitsios, A. (2006). Do investors herd intraday in Australian equities? International Journal of Managerial Finance, 2(3), 196-219.

Hott, C. (2009). Herding behavior in asset markets. Journal of Financial Stability, 5, 35-56.

Kristjanpoller, W. y Arenas, T. (2015). El efecto fin de mes en los principales mercados accionarios latinoamericanos. Contaduría y Administración, 60(1), 53-86. 
Lao, P. y Singh, H. (2011). Herding behaviour in the Chinese and Indian stock markets. Journal of Asian Economics, 22 , 495-506.

Liu S. (2013). An empirical analysis of herd behavior in the Singapore stock market [tesis de maestría en finanzas], Saint Mary's University.

Newey, W. K. y West, K. D. (1987). A simple, positive semi-definite, heteroskedasticity and autocorrelation consistent covariance matrix. Econometrica, 55, 703-708.

Ohlson P. (2010). Herd Behavior on the Swedish Stock Exchange [master thesis in finance], Jônkôping International Business School, Jônkôping.

Ortiz, C., Sarto, J. L. y Vicente, L. (2013). Herding behaviour in Spanish global funds' country allocations. Revista Española de Financiación y Contabilidad, 42(157), 63-81.

Ouarda, M., Bouri, A. E. y Bernard, O. (2013). Herding behavior under markets condition: Empirical evidence on the European financial markets. International Journal of Economics and Financial Issues, 3(1), 214-228.

Scharfstein, D. S. y Stein, J. C. (1990). Herd behavior and investment. The American Economic Review, 80(3), 465-479.

Shiller, R. J. (2003). From efficient markets theory to behavioral finance. Journal of Economic Perspectives, 17(1), 83-104.

Tan, L., Chiang, T. C., Mason, J. R. y Nelling, E. (2008). Herding behavior in Chinese stock markets: An examination of A and B shares. Pacific-Basin Finance Journal, 16, 61-77. 